\title{
Renormalization conjecture and rigidity theory for circle diffeomorphisms with breaks
}

\author{
Konstantin Khanin ${ }^{1 *}$ and Saša Kocič ${ }^{2 \dagger}$ \\ ${ }^{1}$ Dept. of Math., University of Toronto, 40 St. George St., Toronto, ON, Canada M5S 2E4 \\ 2 Dept. of Math., University of Mississippi, P. O. Box 1848, University, MS 38677-1848, USA
}

April 17, 2012

\begin{abstract}
We prove the renormalization conjecture for circle diffeomorphisms with breaks, i.e. that the renormalizations of any two $C^{2+\alpha}$-smooth circle diffeomorphisms with a break point, with the same irrational rotation number and the same size of the break, approach each other exponentially fast in the $C^{2}$-topology. As a corollary, we obtain the following rigidity result: for almost all irrational rotation numbers, any two circle diffeomorphisms with a break, with the same rotation number and the same size of the break, are $C^{1}$-smoothly conjugate to each other.
\end{abstract}

\section{Introduction and statement of the results}

This paper presents the renormalization and rigidity theory for circle diffeomorphisms with a single singular point where the derivative has a jump discontinuity. We call such maps circle diffeomorphisms with breaks and these points the break points. Our first result is the theorem on the exponential convergence of renormalizations for circle maps with breaks provided that they have the same irrational rotation number and the same size of the break, i.e. the square root of the ratio of the derivatives at the break point. More precisely, for any given $\alpha \in(0,1)$ and $c \in \mathbb{R}^{+} \backslash\{1\}$, we prove the following.

*Email: khanin@math.utoronto.ca

†Email: kocic@math.utexas.edu 
Theorem 1.1 There exists $\mu \in(0,1)$, such that for every two $C^{2+\alpha}$-smooth circle diffeomorphisms with a break $T$ and $\widetilde{T}$, with the same irrational rotation number $\rho \in(0,1)$, and the same size of the break $c$, there exists $\underset{\widetilde{T}}{C}>0$, such that the renormalizations $f_{n}$ and $\widetilde{f}_{n}$ of $T$ and $\widetilde{T}$, respectively, satisfy $\left\|f_{n}-\widetilde{f}_{n}\right\|_{C^{2}} \leq C \mu^{n}$, for all $n \in \mathbb{N}$.

We emphasize that the convergence result holds for all irrational rotation numbers and that the exponential rate of convergence is universal, i.e. independent of the maps and, in particular, their rotation numbers, as long as the size of their breaks is the same. This theorem is the final result in the renormalization program for circle diffeomorphisms with a break. As we have previously shown in [15], it implies a strong rigidity statement for such maps. Therefore, as a corollary of Theorem 1.1, we obtain

Theorem 1.2 For almost all irrational rotation numbers $\rho$, any two $C^{2+\alpha}$-smooth circle maps with a break $T$ and $\widetilde{T}$, with the same rotation number $\rho$, and the same size of the break c, are $C^{1}$-smoothly conjugate to each other.

Our main results solve the 20 year-old problem of rigidity for circle diffeomorphisms with breaks. They can be also considered a natural extension of Herman's theory [11], i.e. rigidity theory for circle diffeomorphisms.

To explain how maps with breaks appear naturally in the context of one-dimensional dynamics, we start with a rigid rotation by an angle $\rho$ on a unit circle $\mathbb{T}^{1}$, i.e. a linear map $R: x \mapsto x+\rho$. It is well known that such a map can be regarded as an interval exchange transformation (IET) of two intervals. While in the case of an IET the intervals are transformed by isometries, it is quite natural to consider nonlinear IETs, where the maps acting on the intervals are assumed to be smooth and strictly monotone. In the case of two intervals there are just two branches $h_{1}:[0, \xi] \rightarrow\left[h_{1}(0), 1\right]$ and $h_{2}:[\xi, 1] \rightarrow\left[0, h_{2}(1)\right]$, $\xi \in(0,1)$, with the requirement $h_{1}(0)=h_{2}(1) \in(0,1)$. By matching the derivatives $\left(h_{1}\right)_{+}^{\prime}(0)=\left(h_{2}\right)_{-}^{\prime}(1),\left(h_{1}\right)_{-}^{\prime}(\xi)=\left(h_{2}\right)_{+}^{\prime}(\xi)$, we obtain a circle diffeomorphism $T$ with a lift whose restrictions to $[0, \xi]$ and $[\xi, 1]$ are given by $h_{1}$ and $h_{2}+1$, respectively. This condition is, however, rather artificial in the setting of nonlinear IETs. Without the derivative-matching condition one obtains a circle diffeomorphism $T$ with two break points at $x_{b r}^{(1)}=0$ and $x_{b r}^{(2)}=\xi$. Since the two break points belong to the same orbit of $T$, i.e. $T x_{b r}^{(2)}=x_{b r}^{(1)}$, one can piecewise-smoothly conjugate $T$ to a circle map with a single break point. A natural question to ask is when are two maps of this type smoothly, or piecewise-smoothly, conjugate to each other. This question has been settled by the theory presented in this paper. In a sense this theory is a one-parameter extension of Herman's theory where the break size $c$ plays the role of the parameter. While in the case of circle diffeomorphisms, corresponding to $c=1$, renormalizations converge to a one-dimensional space of linear maps with derivative 1 , in the case $c \neq 1$, the renormalizations converge to a two-dimensional space of fractional linear transformations with very non-trivial dynamics on the limiting attractor. Our main results (Theorem 1.1 and Theorem 1.2) correspond to 
the non-linearizable case of nonlinear IETs of two intervals. The linearizable case of more general IETs has recently been considered by Marmi, Moussa and Yoccoz in [26]. The special case of cyclic permutations, which corresponds to circle maps with more than one point of break and with the product of the sizes of breaks being equal to 1, was considered by Chunha and Smania [4]. In their case, the renormalizations converge to piecewise-affine (linear) maps, rather than fractional linear ones. Our result above (Theorem 1.2) is so far the only result in the general non-affine case.

In the above construction of a nonlinear IET, we respected the special role of the interval $[0,1]$, which is not very natural on the circle. In general, the restriction of the lift $\mathcal{H}$ of such a circle homeomorphism to $[0,1]$ is given by $\mathcal{H}_{1}:[0, \xi] \rightarrow\left[\mathcal{H}_{1}(0), \mathcal{H}_{1}(\xi)\right]$, $\mathcal{H}_{2}:[\xi, 1] \rightarrow\left[\mathcal{H}_{1}(\xi), \mathcal{H}_{1}(0)+1\right]$ and the corresponding circle map will have two break points $x_{b r}^{(1)}=0$ and $x_{b r}^{(2)}=\xi$ which do not necessarily belong to the same orbit. Renormalization and rigidity theory for such maps is currently an open problem.

In the remaining part of this introduction, we provide more details about our results in the historical context of renormalization and rigidity theory in circle dynamics and explain the main difficulty of the problem that we solved. The development of renormalization methods in dynamics started with the work of Feigenbaum $[7,8]$ and Coullet and Tresser [6], on metric universality in period-doubling bifurcations in one-parameter families of one-dimensional maps. It has soon become clear that the universal properties of the dynamics of one-dimensional systems can be understood by studying an associated infinite-dimensional dynamical system: a renormalization operator $\mathcal{R}$ acting on a functional space of the original systems. Typically, the action of the renormalization operator separates the systems into different universality classes, according to their approach to different attractors. As shown by continuous efforts of Sullivan [30], McMullen [27] and Lyubich [24], this Feigenbaum-Coullet-Tresser universality follows from the existence of a hyperbolic fixed point on a space of such maps, with one unstable direction. The theory was extended to infinitely renormalizable unimodal maps of other combinatorial types. The idea of renormalization originally came from statistical mechanics, where it provided an explanation for critical phenomena, by classifying systems into different universality classes, according to their scaling limits and corresponding critical exponents. In dynamics, apart from providing an explanation for the universality of infinitely renormalizable unimodal maps, renormalization methods have also led to advances in rigidity theory [4, 5, 13-19, 29], complex dynamics [27, 30], KAM (Kolmogorov-Arnol'd-Moser) theory [20-22], break-up of invariant tori $[1,25]$, and the reducibility of cocycles and skew-product flows $[3,23]$.

In circle dynamics, the behavior of renormalizations plays a crucial role in proving global rigidity results. Rigidity is the phenomenon of smooth conjugacy between any two maps within a topological equivalence class. The first rigidity result concerned circle diffeomorphisms. For sufficiently-smooth diffeomorphisms, the topological equivalence classes are defined uniquely by a rotation number (Denjoy's lemma). Arnol'd proved the 
first local rigidity result [2], i.e. that every analytic circle diffeomorphism with a Diophantine rotation number, sufficiently close to the rigid rotation, is $C^{1}$-smoothly conjugate to it. He also conjectured that the claim holds true if the assumption of closeness to a rotation is removed. This global rigidity result was proved by Herman [11] (see also [12,17,29,32]). One can show that the renormalizations of any two $C^{2+\alpha}$-smooth circle diffeomorphisms with the same irrational rotation number approach each other exponentially fast. This result implies rigidity, i.e. $C^{1}$-smooth conjugacy between them, only under an additional assumption that the rotation number is Diophantine. The action of the renormalization operator can also be considered on a larger space, involving not only circle diffeomorphisms but also circle diffeomorphisms with a singular point where the derivative vanishes (critical circle maps) or has jump discontinuity (circle maps with a break). It has been conjectured that the renormalizations of any two $C^{2+\alpha}$-smooth circle maps in that space, with the same irrational rotation number and the same type of singularity, approach each other exponentially fast. Despite serious efforts, in the case of critical circle maps this conjecture is still open. In the analytic category, an analogous claim is indeed true, as was proved by de Faria and de Melo [9,10], for bounded-type irrational rotation numbers, and extended to all irrational rotation numbers by Yampolsky [31]. The type of singularity in the case of critical circle maps is characterized by the order of the critical point. For analytic critical circle maps the result holds for all odd-integer orders of the critical point. It is expected, however, that the renormalization conjecture holds for all orders of the critical point (not necessarily integers). For $C^{2+\alpha}$-smooth critical circle maps, it was shown by Khanin and Teplinsky [16] that a proof of the renormalization conjecture would imply robust rigidity, i.e. rigidity for all irrational rotation numbers.

In this paper, we prove the renormalization conjecture for circle maps with a break. It has been known for more than two decades, that the renormalizations of circle maps with a break approach a family of fractional linear transformations [19]. Though significant progress has been made in understanding the dynamics of the renormalization operator on the space of fractional linear transformations [18], any analysis beyond this subset [13, 18] has so far been restricted to those rotation numbers for which one has a bounded geometry, i.e. the case where nearby elements of the dynamical partitions of the circle are of comparable length. Consequently, in this case, the longest elements of the dynamical partitions cannot decrease faster than exponentially (with the renormalization step). This bounded geometry is also characteristic of the case of critical circle maps. The main difficulty of the problem at hand is that one really needs to deal with unbounded geometry. For circle maps with a break, the longest intervals of the dynamical partitions of the circle can decrease at an arbitrary rate. Nevertheless, as we prove in this paper, all such maps with the same irrational rotation number and the same size of the break belong to the same universality class.

In spite of full universality, the problem of unbounded geometry prevents one from obtaining robust rigidity, in the case of circle maps with a break. Indeed, as we proved 
in [14], if the largest elements of dynamical partitions decrease sufficiently rapidly, one can find examples of circle maps with a break of the same size, in the same topological conjugacy class, for which no conjugacy is even Lipschitz continuous. Nevertheless, Theorem 1.1 indeed implies rigidity for almost all irrational rotation numbers (Theorem 1.2). For those rotation numbers, the geometry is super-exponentially bounded, i.e. the logarithms of the ratios of the nearby elements of dynamical partition are bounded by an exponential function.

The paper is organized as follows. In Section 2, we define the renormalizations of circle maps and provide basic definitions and earlier results that we use. In Section 3, we prove general estimates of the renormalization parameters, including the parameter $a_{n}$ (the ratio of the lengths of successive renormalization segments). In Section 4, we define the strings of renormalizations with large $a_{n}$ tails, and obtain a result on the closeness of renormalizations in the tail to fractional linear maps with the same (associated) rotation number. In Section 5, we show that renormalizations with small parameters $a_{n}$ are also close to fractional linear maps with the same rotation number. In Section 6 , we prove an almost commuting property of the renormalization operator and a projection operator onto the space of fractional linear transformations. Finally, in Section 7, we prove Theorem 1.1.

\section{Preliminaries}

\subsection{Renormalization of commuting pairs}

For every orientation-preserving homeomorphism $T$ of the circle $\mathbb{T}^{1}=\mathbb{R} \backslash \mathbb{Z}$ there is a unique rotation number $\rho=\lim _{n \rightarrow \infty} \mathcal{T}^{n}(x) / n \bmod 1$, where $\mathcal{T}$ is a lift of $T$ to $\mathbb{R}$. If $\rho \in(0,1)$ is irrational, it can be expressed uniquely as an infinite continued fraction expansion

$$
\rho=\left[k_{1}, k_{2}, k_{3}, \ldots\right]=\frac{1}{k_{1}+\frac{1}{k_{2}+\frac{1}{k_{3}+\ldots}}},
$$

where $k_{n} \in \mathbb{N}$. Conversely, every infinite sequence of partial quotients $k_{n}$ defines uniquely an irrational number $\rho$ as the limit of the sequence of rational convergents $p_{n} / q_{n}=$ $\left[k_{1}, k_{2}, \ldots, k_{n}\right]$. The denominators satisfy the recursion relation $q_{n+1}=k_{n+1} q_{n}+q_{n-1}$, with $q_{0}=1$ and $q_{-1}=0$.

To define the renormalizations, we start with a marked point $x_{0} \in \mathbb{T}^{1}$, and consider the marked trajectory $x_{i}=T^{i} x_{0}$, with $i \geq 0$. The subsequence $x_{q_{n}}, n \geq 0$, indexed by the denominators of the sequence of rational convergents of the rotation number $\rho$, will be called the sequence of dynamical convergents. We define $x_{q_{-1}}=x_{0}-1$. The combinatorial equivalence of all circle homeomorphisms with the same irrational rotation number implies that the order of the dynamical convergents of $T$ is the same as the order of the dynamical 
convergents for the rigid rotation $R_{\rho}$. The well-known arithmetic properties of the rational convergents now imply that dynamical convergents alternate their order in the following way:

$$
x_{q_{-1}}<x_{q_{1}}<x_{q_{3}}<\cdots<x_{0}<\cdots<x_{q_{2}}<x_{q_{0}} .
$$

The intervals $\left[x_{q_{n}}, x_{0}\right]$, for $n$ odd, and $\left[x_{0}, x_{q_{n}}\right]$, for $n$ even, will be denoted by $\Delta_{0}^{(n)}$, and called the $n$-th renormalization segments. The $n$-th renormalization segment associated to the marked point $x_{i}$ will be denoted by $\Delta_{i}^{(n)}$. The intervals $\Delta_{i}^{(n-1)}=T^{i}\left(\Delta_{0}^{(n-1)}\right)$, for $i=0, \ldots, q_{n}-1$ and $\Delta_{i}^{(n)}=T^{i}\left(\Delta_{0}^{(n)}\right)$, for $i=0, \ldots, q_{n-1}-1$, cover the whole circle without overlapping except at end points and, thus, form the $n$-th dynamical partition $\mathcal{P}_{n}$ of the circle. The first return map on the interval $\Delta_{0}^{(n-1)} \cup \Delta_{0}^{(n)}$ is given by $T^{q_{n}}$ restricted to $\Delta_{0}^{(n-1)}$ and $T^{q_{n-1}}$ restricted to $\Delta_{0}^{(n)}$. The $n$-th renormalization of an orientation-preserving homeomorphism $T$ of the circle $\mathbb{T}^{1}$, with a rotation number $\rho=\left[k_{1}, k_{2}, k_{3}, \ldots\right]$, with respect to the marked point $x_{0} \in \mathbb{T}^{1}$, is given by a pair of functions $\left(f_{n}, g_{n}\right)$, obtained by rescaling the first return map, i.e.

$$
f_{n}=\tau_{n} \circ T^{q_{n}} \circ \tau_{n}^{-1}, \quad g_{n}=\tau_{n} \circ T^{q_{n-1}} \circ \tau_{n}^{-1} .
$$

Here, $\tau_{n}$ is the affine change of coordinates that maps $x_{q_{n-1}}$ to -1 and $x_{0}$ to 0 . Thus, $f_{n}:[-1,0] \rightarrow \mathbb{R}$, and $g_{n}:\left[0, a_{n}\right] \rightarrow \mathbb{R}$, where $a_{n}=\tau_{n}\left(x_{q_{n}}\right)$. The sequence of renormalizations $\left(f_{n}, g_{n}\right)$ can also be generated by the action of a renormalization operator on a space of commuting pairs. Renormalization of commuting pairs was first introduced in [28]. A commuting pair is a pair $(f, g)$ of two real-valued, continuous and strictly-increasing functions $f$ and $g$, with $f(0) \geq 0$ and $g(0) \leq 0$, defined on $[g(0), 0]$ and $[0, f(0)]$, respectively, satisfying $f(g(0))=g(f(0))$. If $g(0)=-1$, the commuting pair is called normalized. If $(f, g)$ is a commuting pair with $g(0)<0$, then $\overline{(f, g)}=\left(\tau \circ f \circ \tau^{-1}, \tau \circ g \circ \tau^{-1}\right)$ with $\tau(z)=-z / g(0)$ is a normalized commuting pair. A commuting pair is nondegenerate if $f(0)>0$. For a normalized, non-degenerate pair $(f, g)$, we define the height $k \in \mathbb{N}_{0}=\mathbb{N} \cup\{0\}$ by the condition $f^{k}(-1) \leq 0<f^{k+1}(-1)$. On a set of renormalizable commuting pairs, i.e. commuting pairs with finite and nonzero height, we define a renormalization operator as $\mathcal{R}(f, g)=\overline{\left(f^{k} \circ g, f\right)}$. Pairs which are not renormalizable are called non-renormalizable.

A pair $(f, g)$ is called infinitely-renormalizable if $\mathcal{R}^{n}(f, g)$ is renormalizable for all $n \in \mathcal{N}_{0}$. Clearly, if the rotation number of $T$ is irrational, then $\mathcal{R}\left(f_{n}, g_{n}\right)=\left(f_{n+1}, g_{n+1}\right)$, for all $n \in \mathbb{N}_{0}$. Here, $\left(f_{0}, g_{0}\right)=\left(\left.\mathcal{T}\right|_{[-1,0]}, \mathcal{I}\right)$, where $\left.\mathcal{T}\right|_{[-1,0]}$ is the restriction of a lift $\mathcal{T}$ of $T$ satisfying $\mathcal{T}(0) \in(0,1]$ to $[-1,0]$ and $\mathcal{I}$ is the identity defined at 0 only.

For normalized pairs $(f, g)$ such that $f(-1)<0$, we define a rotation number $\rho(f, g) \in$ $[0,1]$, by substituting its consecutive heights for partial quotients in the continued fraction expansion $\rho(f, g)=\left[k_{1}, k_{2}, \ldots\right]$, where $k_{n}$ is the height of $\mathcal{R}^{n-1}(f, g)$ (the symbol " $\infty$ " is the terminator of the sequence). On the set of rotation numbers, the renormalization operator acts as Gauss map: $\mathcal{G}\left[k_{1}, k_{2}, \ldots\right]=\left[k_{2}, \ldots\right]$, i.e. $\rho(\mathcal{R}(f, g))=\mathcal{G} \rho(f, g)$. 


\subsection{Definitions and earlier results for circle diffeomorphisms with breaks}

In this paper, we consider renormalizations of $C^{2+\alpha}$-smooth circle diffeomorphisms with breaks, for $\alpha \in(0,1)$, i.e. homeomorphisms $T: \mathbb{T}^{1} \rightarrow \mathbb{T}^{1}$ for which there exists a point $x_{b r} \in \mathbb{T}^{1}$ such that: (i) $T$ is $C^{2+\alpha}$ smooth on $\mathbb{T}^{1} \backslash\left\{x_{b r}\right\}$; (ii) $\inf _{x \neq x_{b r}} T^{\prime}(x)>0$; and (iii) there exist one-sided derivatives $T_{-}^{\prime}\left(x_{b r}\right) \neq T_{+}^{\prime}\left(x_{b r}\right)$. We refer to $x_{b r}$ as the break point and

$$
c=\sqrt{\frac{T_{-}^{\prime}\left(x_{b r}\right)}{T_{+}^{\prime}\left(x_{b r}\right)}} \neq 1
$$

as the size of the break. In the case of circle diffeomorphisms with a break, we will use the break point $x_{b r}$ as the marked point $x_{0}$. One can verify that renormalizations of circle diffeomorphisms with a break of size $c$ satisfy the condition $c_{n}^{2}=\frac{f_{n}^{\prime}(0) g_{n}^{\prime}\left(f_{n}(0)\right)}{g_{n}^{\prime}(0) f_{n}^{\prime}(-1)}$, where $c_{n}=c$ if $n$ is even, $c_{n}=c^{-1}$ if $n$ is odd.

We refer to commuting pairs $(f, g)$ satisfying $c=\sqrt{\frac{f^{\prime}(0) g^{\prime}(f(0))}{g^{\prime}(0) f^{\prime}(-1)}} \in \mathbb{R}_{+} \backslash\{1\}$ as the commuting pairs with break of size $c$. For the renormalization operator acting on commuting pairs $(f, g)$ with breaks of the size $c$, we sometimes write $\mathcal{R}_{c}$ instead of $\mathcal{R}$. Notice that the renormalization operator maps renormalizable commuting pairs with break of size $c$ to commuting pairs with break of size $c^{-1}$.

It is well known that renormalization maps $f_{n}$ and $g_{n}$ for circle diffeomorphisms with a break of size $c \in \mathbb{R}_{+} \backslash\{1\}$ approach, exponentially fast, two particular families of fractional linear transformations

$$
F_{a_{n}, v_{n}, c_{n}}(z)=\frac{a_{n}+c_{n} z}{1-v_{n} z}, \quad G_{a_{n}, v_{n}, c_{n}}(z)=\frac{-c_{n}+z}{c_{n}-\frac{c_{n}-1-v_{n}}{a_{n}} z},
$$

with

$$
a_{n}=\frac{\left|\Delta_{0}^{(n)}\right|}{\left|\Delta_{0}^{(n-1)}\right|}, \quad v_{n}=\frac{c_{n}-a_{n}-b_{n}}{b_{n}}, \quad b_{n}=\frac{\left|\Delta_{0}^{(n-1)}\right|-\left|\Delta_{q_{n-1}}^{(n)}\right|}{\left|\Delta_{0}^{(n-1)}\right|} .
$$

We will often abbreviate the notation by writing $F_{n}=F_{a_{n}, v_{n}, c_{n}}$ and $G_{n}=G_{a_{n}, v_{n}, c_{n}}$. The derivatives of these maps are given by

$$
F_{a, v, c}^{\prime}(z)=\frac{c+a v}{(1-v z)^{2}}, \quad G_{a, v, c}^{\prime}(z)=\frac{c\left(1-\frac{c-1-v}{a}\right)}{\left(c-\frac{c-1-v}{a} z\right)^{2}} .
$$

It is easy to see that $V=\operatorname{Var}_{\mathbb{T}^{1}} \ln T^{\prime}<\infty$. It follows that the map $T$ satisfies the Denjoy's lemma [19], which implies that $\left|\ln \left(T^{q_{n}}\right)^{\prime}(x)\right| \leq V$, for all $x \in \mathbb{T}^{1}$. In particular, we have

(A) $\left|\ln f_{n}^{\prime}(x)\right| \leq V$, for all $x \in[-1,0]$ (at the end points, both the left and right derivatives are considered). 


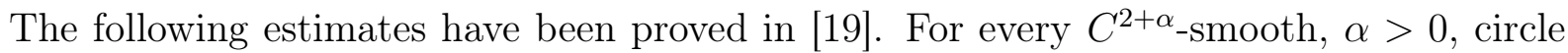
diffeomorphism $T$ with a break of size $c$, there exist constants $\mathcal{C}>0$ and $\lambda \in(0,1)$, such that, for all $n \in \mathbb{N}$, we have

(B) $\left\|f_{n}-F_{a_{n}, v_{n}, c_{n}}\right\|_{C^{2}} \leq \mathcal{C} \lambda^{n},\left\|g_{n}-G_{a_{n}, v_{n}, c_{n}}\right\|_{C^{1}} \leq \mathcal{C} \lambda^{n}$,

(C) $\left|a_{n}+b_{n} M_{n}-c_{n}\right| \leq \mathcal{C} \lambda^{n}$, and

(D) $\left|M_{n+1}-c_{n+1}\left(1+a_{n+1} a_{n}\left(M_{n}-1\right)\right)\right| \leq \mathcal{C} \lambda^{n}$, where

$$
M_{n}=\exp \left((-1)^{n} \int_{\Delta_{0}^{(n-1)}} \frac{\left(T^{q_{n}}\right)^{\prime \prime}(z)}{2\left(T^{q_{n}}\right)^{\prime}(z)} d z\right)=\exp \left((-1)^{n} \sum_{i=0}^{q_{n}-1} \int_{\Delta_{i}^{(n-1)}} \frac{T^{\prime \prime}(z)}{2 T^{\prime}(z)} d z\right) \text {. }
$$

Property (B) is a statement about the approach of renormalization maps to fractional linear transformations. Property $(\mathrm{C})$ is a consequence of the commutation relation of the maps $f_{n}$ and $g_{n}$. Property (D) provides a relation between nonlinearities of the maps $f_{n}$ and $f_{n+1}$.

We also define

$$
N_{n}=\exp \left((-1)^{n} \sum_{i=0}^{q_{n-1}-1} \int_{\Delta_{i}^{(n)}} \frac{T^{\prime \prime}(z)}{2 T^{\prime}(z)} d z\right)
$$

Clearly, $M_{n} N_{n}=\exp \left((-1)^{n} \int_{S^{1}} \frac{T^{\prime \prime}(z)}{2 T^{\prime}(z)} d z\right)=c^{(-1)^{n}}=c_{n}$.

For a normalized commuting pair $(f, g)$ with positive height, we define, as in [18], the canonical lift $H_{f, g}(w): \mathbb{R} \mapsto \mathbb{R}$, satisfying $H_{f, g}(w+1)=H_{f, g}(w)+1$, and

$$
H_{f, g}(w)= \begin{cases}H_{f, g}^{(1)}(w), & w \in\left[-1, \phi\left(f^{-1}(0)\right)\right), \\ 1+H_{f, g}^{(2)}(w), & w \in\left[\phi\left(f^{-1}(0)\right), 0\right),\end{cases}
$$

where

$$
H_{f, g}^{(1)}(w)=\phi \circ f \circ \phi^{-1}, \quad H_{f, g}^{(2)}(w)=\phi \circ g \circ f \circ \phi^{-1},
$$

and $\phi:[-1, f(0)] \rightarrow \mathbb{R}$ is the fractional linear transformation that maps $(-1,0, f(0))$ into $(-1,0,1)$, i.e.

$$
\phi(z)=\frac{(f(0)+1) z}{2 f(0)+(f(0)-1) z} .
$$

The derivative of the latter coordinate transformation is given by

$$
\phi^{\prime}(z)=\frac{2 f(0)(f(0)+1)}{(2 f(0)+(f(0)-1) z)^{2}} .
$$


Notice that the canonical lift $H_{f, g}$ of a commuting pair with a break of size $c$ has, in general, two break points. Nevertheless, they belong to the same orbit and the product of the sizes of their breaks is equal to $c$.

We will identify each point $(a, v) \in \mathbb{R}^{2}$ with the corresponding pair of fractional linear maps $\left(F_{a, v, c}, G_{a, v, c}\right)$, whenever the latter is well-defined. The following sets play an important role in the renormalization of commuting pairs of fractional linear transformations. We define

$$
\mathcal{D}_{c}=\left\{(a, v): 1 / 2 \leq \frac{v}{c-1}<1, \frac{c(c-v-1)}{v} \leq a \leq c\right\},
$$

and $\check{\mathcal{D}}_{c}=\mathcal{D}_{c} \cap\left\{(a, v): a>(c-1)^{2} / 4 v\right\}$, if $c>1$, and $\check{\mathcal{D}}_{c}=\mathcal{D}_{c} \cap\{(a, v): v>$ $\left.a(c-1)^{2} / 4 c+c-1\right\}$, if $c<1$. Some of the estimates for the renormalizations of pairs of fractional linear transformations by Khanin and Teplinsky [18] are important for us. It was shown therein that the renormalization operator maps all infinitely renormalizable pairs in $\check{\mathcal{D}}_{c}$ into $\check{\mathcal{D}}_{1 / c}$. Moreover, these sets are absorbing areas for the dynamics of the renormalization operator on a space of commuting pairs of fractional linear maps, i.e. each infinitely renormalizable commuting pair of fractional linear maps eventually falls inside these sets, under the action of the renormalization operator $\mathcal{R}$. The set of points in $\{(a, v): 0<a \leq c, a+v-c+1>0\} \supset \check{\mathcal{D}}_{c}$ with the same irrational rotation number $\rho \in(0,1)$ is a continuous curve $a=\gamma_{\rho, c}(v), v>-1$, such that the slope of any secant line, in the $(v, a)$ coordinate system, belongs to the interval $(-1,0)$. We will refer to this slope as the slope of the curve $\gamma_{\rho, c}$. Furthermore, for $c>1$ and all irrational $\rho \in(0,1)$, all curves $\gamma_{\rho, c}$ lie above the hyperbola $a=\frac{(c-1)^{2}}{4 v}$.

We end this section with some comments about the notation. We write $A_{n}=\Theta\left(B_{n}\right)$, if there exits a constant $K_{1}>0$, such that $K_{1}^{-1} B_{n} \leq A_{n} \leq K_{1} B_{n}$, for all $n$. We write $A_{n}=\mathcal{O}\left(B_{n}\right)$, if there exists a constant $K_{2} \in \mathbb{R}$, such that $-K_{2} B_{n} \leq A_{n} \leq K_{2} B_{n}$, for all $n$.

\section{A priori estimates of the renormalization parameters}

Proposition 3.1 $M_{n}=v_{n}+1+\mathcal{O}\left(\lambda^{n}\right)$ and $\ln M_{n}=\mathcal{O}(1)$, for all $n \in \mathbb{N}$.

It follows from (A) that $f_{n}^{\prime}(z)=\left(T^{q_{n}}\right)^{\prime}\left(\tau_{n}^{-1}(z)\right)$ is bounded both from above and below by positive constants. The same is true for $F_{n}^{\prime}(z)=f_{n}^{\prime}(z)+\mathcal{O}\left(\lambda^{n}\right)$, for sufficiently large $n$. In particular, this implies $v_{n} \geq-1$, for sufficiently large $n$, as the opposite inequality would lead to $F_{n}^{\prime}(0)=c_{n}+a_{n} v_{n}<c_{n}-a_{n}=\left(v_{n}+1\right) b_{n}<0$. Since $\frac{f_{n}^{\prime}(0)}{f_{n}^{\prime}(-1)}=\frac{F_{n}^{\prime}(0)+\mathcal{O}\left(\lambda^{n}\right)}{F_{n}^{\prime}(-1)+\mathcal{O}\left(\lambda^{n}\right)}=$ $\left(1+v_{n}\right)^{2}+\mathcal{O}\left(\lambda^{n}\right), 1+v_{n}$ is also bounded from above and below by positive constants. 
The first claim follows from the identity

$$
M_{n}=\exp \left(\frac{1}{2} \int_{x_{q_{n-1}}}^{x_{0}}\left(\ln \left(T^{q_{n}}\right)^{\prime}(z)\right)^{\prime} d z\right)=\sqrt{\frac{\left(T^{q_{n}}\right)^{\prime}(0)}{\left(T^{q_{n}}\right)^{\prime}(-1)}}=\sqrt{\frac{f_{n}^{\prime}(0)}{f_{n}^{\prime}(-1)}} .
$$

The second claim follows from (2.8), the fact that $T^{\prime}$ is bounded from below by a positive constant and the fact that $T^{\prime \prime}$ is bounded.

Proposition 3.2 There exists $\epsilon>0$ such that, for $n$ sufficiently large, if $c_{n}>1$, then $a_{n} \in\left(\epsilon, c_{n}-\Theta\left(a_{n+1}\right)\right)$; if $c_{n}<1$, then $a_{n} \in\left(0, c_{n}-\epsilon\right)$;

Proof. It follows from Proposition 3.1 that $c_{n}-a_{n}=\left(v_{n}+1\right) b_{n}=\Theta\left(b_{n}\right)$ and, in particular, $a_{n}$ is bounded. In the case $c_{n}>1$, assume that $a_{n}$ is very small. Due to (B), for sufficiently large $n, f_{n}^{\prime}(0)$ is close to $F_{n}^{\prime}(0)=c_{n}+a_{n} v_{n}$, which is close to $c_{n}>1$. Also, $f_{n}^{\prime \prime}(z)$ is close to $F_{n}^{\prime \prime}(z)=2 v_{n} \frac{c_{n}+a_{n} v_{n}}{\left(1-v_{n} z\right)^{3}}$, which is bounded due to Proposition 3.1. This implies that $f_{n}(z)=z$ at some point $z \in[-1,0]$, close to 0 , which contradicts the fact that the rotation number of $T$ is irrational.

In the case $c_{n}<1$, assume that $c_{n}-a_{n}$ is very small. Then, $b_{n}$ must be very small. Furthermore, since from the definitions $a_{n+1} a_{n} \leq b_{n}$, and since $a_{n+1}>\epsilon$, for sufficiently large $n$, we obtain that $a_{n}$ must be very small, which contradicts $c_{n}-a_{n}$ being small. QED

Corollary 3.3 There exists $\delta>0$, such that, for sufficiently large $n, a_{n+1} a_{n}<1-\delta$.

Proof. It follows directly from Proposition 3.2, and the fact that $c_{n} c_{n+1}=1 . \quad$ QED

Proposition $3.4 v_{n}=c_{n}-1+\mathcal{O}\left(a_{n}\right)$, for all $n \in \mathbb{N}_{0}$.

Proof. Since $b_{n}=1-a_{n} f_{n-1}^{\prime}(\zeta)$, for some $\zeta \in\left(-a_{n} a_{n-1}, 0\right)$, using property $(B)$, the explicit form of the derivative (2.7), and the fact that $F_{n}^{\prime \prime}$ is bounded, we obtain

$$
b_{n}=1-a_{n}\left(c_{n-1}+a_{n-1} v_{n-1}+\mathcal{O}\left(\lambda^{n}\right)+\mathcal{O}\left(a_{n} a_{n-1}\right)\right) .
$$

For small $a_{n}$, directly from the definition (2.6) of $v_{n}$, we further have

$$
v_{n}=\left(c_{n}-a_{n}\right)\left(1+\mathcal{O}\left(a_{n}\right)\right)-1=c_{n}-1+\mathcal{O}\left(a_{n}\right) .
$$

Since $a_{n}$ is bounded, this proves the claim.

QED

Proposition 3.5 Let $c \in \mathbb{R}_{+} \backslash\{1\}$. There exist a universal constant $\lambda \in(0,1)$, such that the estimates $(B),(C)$ and $(D)$ hold true for every $C^{2+\alpha}$-smooth circle map $T$ with a break of size $c$, and every $n \in \mathbb{N}$. 
Proof. There exist a universal constant $\bar{V}>0$, such that for every $C^{2+\alpha}$-smooth circle map $T$ with a break of size $c$ the following holds. There exists $N_{0} \in \mathbb{N}$, such that for all $n \geq N_{0}$, we have $\left|f_{n}^{\prime}\right| \leq \bar{V}$. This follows from Proposition 3.1 and Proposition 3.2, using property (B). It follows from [29] (see Lemma 2 therein) that there is a universal constant $\lambda=\left(1+e^{-\bar{V}}\right)^{-1 / 2}$, such that $\frac{\left|\Delta_{0}^{(n+2)}\right|}{\left|\Delta_{0}^{(n)}\right|} \leq \lambda^{2}$, for $n \geq N_{0}$. This implies that the estimates (B), (C) and (D) are valid with the same $\lambda$ for every such map $T$, with some $\mathcal{C}>0$ (depending on $T$ ), for every $n \in \mathbb{N}$.

QED

\section{Strings of renormalizations with large $a_{n}$ tails}

We define a string of renormalizations (or simply a string) to be a (finite or infinite) sequence $f_{n}$ with $n \in\left[n_{1}, n_{2}-1\right], n_{1} \in \mathbb{N} \cup\{0\}$ and $n_{2} \in \mathbb{N} \cup\{\infty\}$. We call $n_{2}-n_{1}$, the length of the string. It can be finite or infinite. We choose some positive $\sigma<\epsilon$ from Proposition 3.2, and assume that the strings have tails with (relatively) large $a_{n}$, i.e. for all $n \in\left[n_{1}+1, n_{2}-1\right]$, we have $a_{n}>\sigma \lambda_{1^{-}}^{n}$, for some $\lambda_{1^{-}} \in(\lambda, 1)$. Each finite string ends with $c_{n_{2}}<1$ and $a_{n_{2}} \leq \sigma \lambda_{1^{+}}^{n_{2}}$, for some $\lambda_{1^{+}} \in\left[\lambda_{1^{-}}, 1\right)$. We consider two types of strings: (i) an initial string, starting at some $n_{1}=n_{0} \in \mathbb{N}_{0}$, and (ii) an ordinary string, starting at some $n_{1} \in \mathbb{N}$ with $a_{n_{1}} \leq \sigma \lambda_{1+}^{n_{1}}$. If $\lambda_{1^{+}}>\lambda_{1^{-}}$, there is certain freedom in the choice of strings within the renormalization sequence $f_{n}$. We will use this freedom later on. We assume that the initial string is sufficiently long so that the estimates of the previous section are already valid. Notice that the initial string can be made arbitrary long by taking $\sigma$ sufficiently small and that for an ordinary string we have $a_{n_{1}} \leq \sigma<\epsilon$ and thus $c_{n_{1}}<1$.

The objective of this section is to show that, with an exponentially small (in $n$ ) change of the parameter $a_{n}$ of the fractional linear map $F_{a_{n}, v_{n}, c_{n}}$, one can obtain a fractional linear map with the same rotation number as $\left(f_{n}, g_{n}\right)$, for sufficiently large $n$, in the initial string and for all integer $n \in\left[n_{1}+1, n_{2}-1\right]$, in an ordinary string.

Proposition 4.1 There exists $\epsilon_{1}>0$, such that $\frac{v_{n}}{c_{n}-1} \in\left(\epsilon_{1}, 1-\Theta\left(\lambda_{1^{-}}^{n}\right)\right)$, for sufficiently large $n$, in the initial string, and for all $n \in\left[n_{1}+2, n_{2}-1\right]$ in an ordinary string. In an ordinary string, we also have $\frac{v_{n_{1}}}{c_{n_{1}}-1} \in\left(1-\Theta\left(\lambda_{1^{+}}^{n}\right), 1+\Theta\left(\lambda_{1^{+}}^{n_{1}}\right)\right)$ and $\frac{v_{n_{1}+1}}{c_{n_{1}+1^{-1}}} \in$ $\left(1-\Theta\left(\lambda_{1^{+}}^{n}\right), 1+\Theta\left(\lambda^{n_{1}}\right)\right)$.

Proof. It follows from (D) and Proposition 3.1 that

$$
v_{n+1}=c_{n+1}\left(1+v_{n} a_{n} a_{n+1}\right)-1+\mathcal{O}\left(\lambda^{n}\right) .
$$

Applying this estimate recursively, first for $v_{n+2}$ and then for $v_{n+1}$, we obtain

$$
\frac{v_{n+2}}{c_{n+2}-1}=a_{n+2} a_{n+1} a_{n+1} a_{n} \frac{v_{n}}{c_{n}-1}+\left(1-a_{n+2} a_{n+1}\right)+\mathcal{O}\left(\lambda^{n}\right),
$$


since $c_{n+2}=c_{n}$. It is now easy to see from Corollary 3.3 that if $\frac{v_{n}}{c_{n}-1}$ is negative, in two steps it will increases by a positive constant; once it becomes positive, it will stay larger than a positive constant that can be chosen arbitrarily close to $\delta$. This proves the desired lower bound for sufficiently large $n$ in the initial string, if the string is long enough. For an ordinary string, it follows directly from Proposition 3.4 that $\frac{v_{n_{1}}}{c_{n_{1}}-1}=1+\mathcal{O}\left(\lambda_{1^{+}}^{n_{1}}\right)$. Identity (4.1) implies $\frac{v_{n_{1}+1}}{c_{n_{1}+1}-1}=1-\Theta\left(a_{n_{1}}\right)+\mathcal{O}\left(\lambda^{n_{1}}\right)$. The recursion relation (4.2) leads to the desired lower bound for the remaining $n$ in the string.

By rewriting the equality (4.2) as

$$
1-\frac{v_{n+2}}{c_{n+2}-1}=a_{n+2} a_{n+1}\left[a_{n+1} a_{n}\left(1-\frac{v_{n}}{c_{n}-1}\right)+\left(1-a_{n+1} a_{n}\right)\right]+\mathcal{O}\left(\lambda^{n}\right),
$$

we see that, for sufficiently large $n$ in the initial string, we have $1-\frac{v_{n}}{c_{n}-1}>\Theta\left(\lambda_{1^{-}}^{n}\right)$. If for some $n \in \mathbb{N}_{0}, 1-\frac{v_{n}}{c_{n}-1}<-\delta$, it will increase in two steps by an amount larger than a positive constant and, thus, in a finite number of steps, it will become positive. Once it is positive, it will remain positive for all larger $n$ belonging to the same even or odd subsequence. This proves the desired upper bound for sufficiently large $n$ in the initial string. For an ordinary string, the above estimates on $\frac{v_{n}}{c_{n}-1}$, for $n=n_{1}$ and $n=n_{1}+1$, and the recursive relation (4.3) imply the desired upper bounds.

QED

Proposition 4.2 For sufficiently large $n$ in the initial string and for all $n \in\left[n_{1}, n_{2}-1\right]$ in an ordinary string, we have

$$
a_{n+1} \geq \frac{c_{n+1}\left(c_{n+1}-v_{n+1}-1\right)}{v_{n+1}}+\mathcal{O}\left(\lambda^{n}\right)
$$

Proof. It follows directly from (4.1) that

$$
\frac{v_{n+1}+1-c_{n+1}}{a_{n+1}}=c_{n+1} v_{n} a_{n}+\frac{1}{a_{n+1}} \mathcal{O}\left(\lambda^{n}\right),
$$

and, thus,

$$
\frac{c_{n+1}-v_{n+1}-1}{a_{n+1}}-\frac{v_{n+1}}{c_{n+1}}=c_{n}-1-v_{n} a_{n}\left(a_{n+1}+c_{n+1}\right)+\frac{1}{a_{n+1}} \mathcal{O}\left(\lambda^{n}\right) .
$$

We further have

$$
\left|v_{n}\right| a_{n}\left(c_{n+1}+a_{n+1}\right) \leq\left|v_{n} b_{n}+v_{n} \frac{a_{n}}{c_{n}}\right|=\left|v_{n} \frac{c_{n}-a_{n}}{1+v_{n}}+v_{n} \frac{a_{n}}{c_{n}}\right| .
$$

For $\frac{v_{n}}{c_{n}-1} \in(0,1]$, the right-hand side of $(4.7)$ is smaller or equal to $\left|c_{n}-1\right|$, since it is the absolute value of an increasing function of $v_{n}$, which takes the value $c_{n}-1$ at $v_{n}=c_{n}-1$. Thus, if $c_{n}>1$, we have

$$
\frac{c_{n+1}-v_{n+1}-1}{a_{n+1}}-\frac{v_{n+1}}{c_{n+1}} \geq \frac{1}{a_{n+1}} \mathcal{O}\left(\lambda^{n}\right),
$$


and, since $v_{n+1}$ is bounded from above by a negative constant, by Proposition 4.1, we have (4.4). If $c_{n}<1$, then one gets the same inequality (4.4) for sufficiently large $n$ in the initial string and for $n>n_{1}$ in an ordinary string. For $n=n_{1}$ in an ordinary string, we obtain (4.4) (without the error term) directly from (4.6), using the fact that $v_{n}$ and $a_{n}$ are bounded, as follows from Proposition 3.1 and Proposition 3.2.

QED

Proposition 4.3 The point $\left(a_{n}, v_{n}\right)$ belongs to the $\mathcal{O}\left(\lambda^{n}\right)$-neighborhood of $\check{\mathcal{D}}_{c_{n}}$, for sufficiently large $n$ in the initial string and for all $n \in\left[n_{1}+1, n_{2}-1\right]$ in an ordinary string. If $c_{n}>1$, then $\frac{v_{n}}{c_{n}-1} \in\left(\frac{1}{2}-\Theta\left(\lambda^{n}\right), 1+\Theta\left(\lambda^{n}\right)\right)$. If $c_{n}<1$, then $\frac{v_{n}}{c_{n}-1} \in\left(\frac{1}{2}+\epsilon_{2}, 1-\Theta\left(\lambda_{1^{-}}^{n}\right)\right)$, for some $\epsilon_{2}>0$.

Proof. It follows from Proposition 3.2, Proposition 4.1 and Proposition 4.2 that, if $c_{n}>1$, then we have $\frac{v_{n}}{c_{n}-1} \in\left(\frac{1}{2}-\Theta\left(\lambda^{n}\right), 1+\Theta\left(\lambda^{n}\right)\right)$. If $c_{n}<1$, then $\frac{v_{n}}{c_{n}-1} \in\left(\frac{1}{2}+\epsilon_{2}, 1-\Theta\left(\lambda_{1^{-}}^{n}\right)\right)$. Together with Proposition 3.2 and Proposition 4.2, these estimates show that $\left(a_{n}, v_{n}\right)$ belongs to $\mathcal{O}\left(\lambda^{n}\right)$-neighborhood of $\mathcal{D}_{c_{n}}$ (defined by (2.14)), for sufficiently large $n$ in the initial string and all $n \in\left[n_{1}+1, n_{2}-1\right]$ in an ordinary string.

Let $c_{n}>1$. Since the rotation number is irrational, using (B), for all $z \in[-1,0]$, we have

$$
z<f_{n}(z) \leq F_{n}(z)+\mathcal{O}\left(\lambda^{n}\right) .
$$

In particular, for some $z_{0}=-\frac{c_{n}-1}{2 v_{n}}+\mathcal{O}\left(\lambda^{n}\right) \in(-1,0)$, we obtain

$$
F_{n}\left(z_{0}\right)-z_{0}=\frac{2}{c_{n}+1}\left(a_{n}-\frac{\left(c_{n}-1\right)^{2}}{4 v_{n}}\right)+\mathcal{O}\left(\lambda^{n}\right) \geq \mathcal{O}\left(\lambda^{n}\right),
$$

and, thus,

$$
a_{n}-\frac{\left(c_{n}-1\right)^{2}}{4 v_{n}} \geq \mathcal{O}\left(\lambda^{n}\right) .
$$

This inequality, together with (4.1), implies

$$
\frac{v_{n+1}+1-c_{n+1}}{c_{n+1} a_{n+1}} \geq \frac{\left(c_{n}-1\right)^{2}}{4}+\frac{1}{a_{n+1}} \mathcal{O}\left(\lambda^{n}\right)=\frac{\left(c_{n+1}-1\right)^{2}}{4\left(c_{n+1}\right)^{2}}+\frac{1}{a_{n+1}} \mathcal{O}\left(\lambda^{n}\right) .
$$

The estimates (4.11) and (4.12) show that, for sufficiently large $n$ in the initial string and for all $n \in\left[n_{1}+1, n_{2}-1\right]$ in an ordinary string, $\left(a_{n}, v_{n}\right)$, in fact, belongs to $\mathcal{O}\left(\lambda^{n}\right)$ neighborhood of $\check{\mathcal{D}}_{c_{n}}$.

QED

Proposition 4.4 For sufficiently large $n$ in the initial string and for all $n \in\left[n_{1}+1, n_{2}-1\right]$, we have $v_{n}+a_{n}+1-c_{n}>\Theta\left(a_{n}\right)$. 
Proof. If $c_{n}>1$, then it follows from Proposition 3.2, Proposition 4.2 and Proposition 4.3 that

$$
a_{n}+v_{n}+1-c_{n} \geq a_{n}-a_{n} \frac{v_{n}}{c_{n}}-\mathcal{O}\left(\lambda^{n}\right)>\Theta\left(a_{n}\right) .
$$

If $c_{n}<1$, then it follows from Proposition 4.1 that

$$
a_{n}+v_{n}+1-c_{n}>a_{n}+\Theta\left(\lambda_{1^{-}}^{n}\right)>a_{n} .
$$

QED

Proposition 4.5 There exists a constant $C_{1}>1$, such that for sufficiently large $n$, and every $z$ in the domain of the corresponding function, we have

$$
C_{1}^{-1}<F_{n}^{\prime}(z), G_{n}^{\prime}(z)<C_{1}, \quad C_{1}^{-1} a_{n}<\phi_{n}^{\prime}(z)<\frac{C_{1}}{a_{n}} .
$$

Proof. The bounds on $F_{n}^{\prime}$ and $G_{n}^{\prime}$ follow from properties (A) and (B), as shown at the beginning of the proof of Proposition 3.1. The bound on $\phi_{n}^{\prime}$ is obvious.

QED

Proposition 4.6 There exists $C_{2}>0$, such that $\left|H_{f_{n}, g_{n}}(w)-H_{F_{n}, G_{n}}(w)\right| \leq \frac{C_{2}}{a_{n}} \lambda^{n}$, for sufficiently large $n$ and all $w \in \mathbb{R}$.

Proof. Since $f_{n}$ and $F_{n}$ are monotonically increasing, $f_{n}^{-1}(0)$ and $F_{n}^{-1}(0)$ are defined uniquely. Furthermore, it follows from the fact that the rotation number of $\left(f_{n}, g_{n}\right)$ is irrational, Proposition 3.1 and Proposition 3.2 that $f_{n}^{-1}(0), F_{n}^{-1}(0) \in(-1,0)$. Notice that, since $f_{n}(0)=F_{n}(0), \phi$ is the same for $\left(f_{n}, g_{n}\right)$ and $\left(F_{n}, G_{n}\right)$. On $\left[-1, \min \left\{\phi\left(F_{n}^{-1}(0)\right), \phi\left(f_{n}^{-1}(0)\right)\right\}\right)$, using property $(\mathrm{B})$, we have

$$
\begin{array}{r}
\left|H_{f_{n}, g_{n}}(w)-H_{F_{n}, G_{n}}(w)\right|=\left|\phi \circ f_{n} \circ \phi^{-1}(w)-\phi \circ F_{n} \circ \phi^{-1}(w)\right| \\
=\phi^{\prime}(\zeta)\left|f_{n} \circ \phi^{-1}(w)-F_{n} \circ \phi^{-1}(w)\right| \leq \frac{C_{1}}{a_{n}} \mathcal{C} \lambda^{n},
\end{array}
$$

where $\zeta$ is a point between $f_{n} \circ \phi^{-1}(w)$ and $F_{n} \circ \phi^{-1}(w)$. On $\left[\max \left\{\phi\left(F_{n}^{-1}(0)\right), \phi\left(f_{n}^{-1}(0)\right)\right\}, 0\right)$, we have

$$
\begin{aligned}
\left|H_{f_{n}, g_{n}}(w)-H_{F_{n}, G_{n}}(w)\right| & =\left|\phi \circ g_{n} \circ f_{n} \circ \phi^{-1}(w)-\phi \circ G_{n} \circ F_{n} \circ \phi^{-1}(w)\right| \\
& =\phi^{\prime}\left(\zeta_{1}\right)\left|g_{n} \circ f_{n} \circ \phi^{-1}(w)-G_{n} \circ F_{n} \circ \phi^{-1}(w)\right| \\
& \leq \phi^{\prime}\left(\zeta_{1}\right)\left(G_{n}^{\prime}\left(\zeta_{2}\right)\left|f_{n} \circ \phi^{-1}(w)-F_{n} \circ \phi^{-1}(w)\right|\right. \\
& \left.+\left|\left(g_{n}-G_{n}\right) \circ f_{n} \circ \phi^{-1}(w)\right|\right) \leq \frac{C_{1}}{a_{n}}\left(C_{1}+1\right) \mathcal{C} \lambda^{n} .
\end{aligned}
$$

Here, $\zeta_{1}$ is a point between $g_{n} \circ f_{n} \circ \phi^{-1}(w)$ and $G_{n} \circ F_{n} \circ \phi^{-1}(w)$, and $\zeta_{2}$ is a point between $f_{n} \circ \phi^{-1}(w)$ and $F_{n} \circ \phi^{-1}(w)$. 

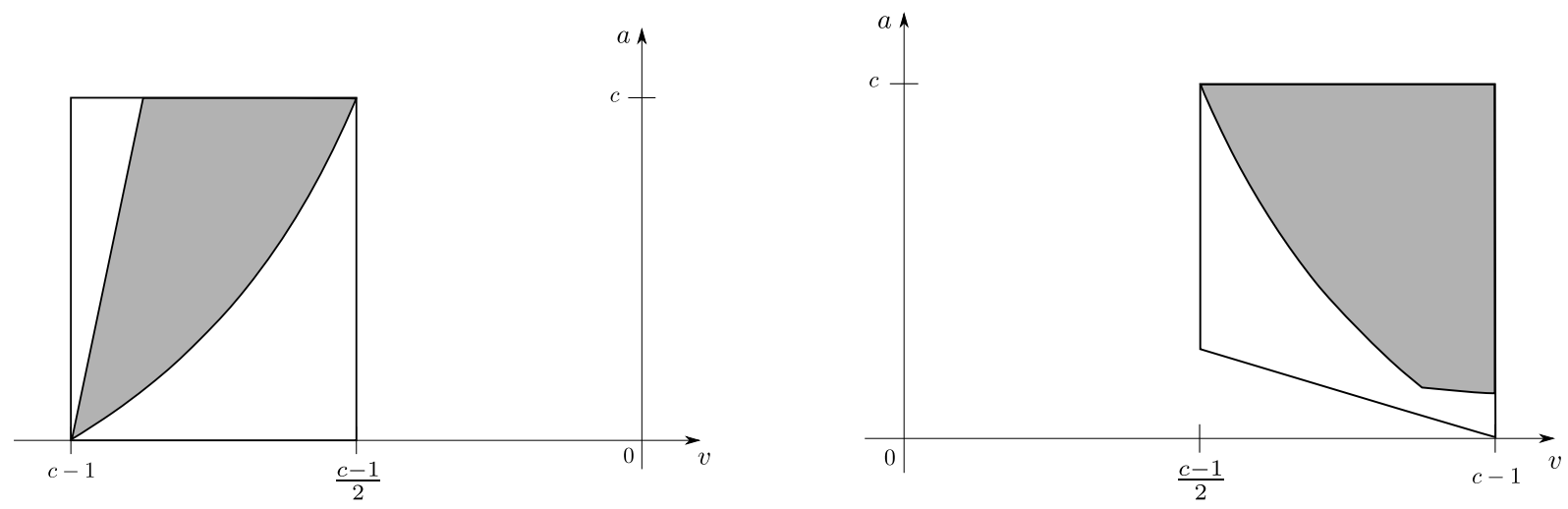

Figure 1: Regions $\check{\mathcal{D}}_{c}$ (shaded) and $\Phi_{c}^{\varepsilon}$ (trapezoid) for $0<c<1$ (left) and $c>1$ (right).

Since the functions $H_{f_{n}, g_{n}}$ and $H_{F_{n}, G_{n}}$ are continuous and monotonically increasing, and since $H_{f_{n}, g_{n}}\left(\phi\left(f_{n}^{-1}(0)\right)\right)=0$ and $H_{F_{n}, G_{n}}\left(\phi\left(F_{n}^{-1}(0)\right)\right)=0$, we obtain a similar estimate

$$
\left|H_{f_{n}, g_{n}}(w)-H_{F_{n}, G_{n}}(w)\right| \leq \frac{C_{1}}{a_{n}}\left(C_{1}+2\right) \mathcal{C} \lambda^{n}
$$

in the interval $\left[\min \left\{\phi\left(F_{n}^{-1}(0)\right), \phi\left(f_{n}^{-1}(0)\right)\right\}, \max \left\{\phi\left(F_{n}^{-1}(0)\right), \phi\left(f_{n}^{-1}(0)\right)\right\}\right]$. Thus, on the whole interval $[-1,0]$, we have the desired estimate, and the claim follows.

QED

Let $\varepsilon \geq 0$ and $\varsigma>0$ be given. If $c>1$, we define

$$
\Phi_{c}^{\varepsilon}=\left\{(a, v): \varepsilon<a<c, \frac{1}{2}-\varsigma \lambda^{n}<\frac{v}{c-1}<1+\varsigma \lambda^{n}, v+a+1-c>\varepsilon\right\} .
$$

If $c<1$, we define

$$
\Phi_{c}^{\varepsilon}=\left\{(a, v): \varepsilon \lambda_{1^{-}}^{n}<a<c-\varepsilon, \frac{1}{2}+\varepsilon<\frac{v}{c-1}<1-\varepsilon \lambda_{1^{-}}^{n}\right\} .
$$

Proposition 3.2, Proposition 4.3, and Proposition 4.4 show that there exist $\varepsilon>0$ and $\varsigma>0$ such that, for sufficiently large $n$ in the initial string and for all $n \in\left[n_{1}+1, n_{2}-1\right]$ in an ordinary string $\left(a_{n}, v_{n}\right)$ belongs to $\Phi_{c_{n}}^{\varepsilon}$. In the following, we assume that $\varepsilon$ and $\varsigma$ have been chosen such that this is the case. We abbreviate the notation $H_{n}=H_{F_{n}, G_{n}}$. The value that $H_{n}$ takes at point $w$ will be denoted by $H_{n}\left(w ; a_{n}, v_{n}\right)$, when necessary to specify the parameters of $F_{n}$ and $G_{n}$.

Proposition 4.7 There exists $\mu_{c_{n}, \varepsilon}>0$ such that, for any $\left(a_{n}, v_{n}\right) \in \Phi_{c_{n}}^{\varepsilon}$, we have $\frac{\partial H_{n}^{(i)}\left(w ; a_{n}, v_{n}\right)}{\partial a_{n}} \geq \mu_{c_{n}, \varepsilon} a_{n}^{5}$, for $i=1,2$. 
Proof. To abbreviate the notation, let us write $a, v, c$ instead of $a_{n}, v_{n}, c_{n}$, respectively. A direct calculation gives us that $\frac{\partial H_{n}^{(1)}}{\partial a}=-4 w P_{1}(w) / Q_{1}^{2}(w)$, for $w \in\left[-1,-\frac{a+1}{2 c+1-a}\right]$, where $P_{1}(w)=-\left(2 v c+2 a c+v+v a^{2}-2 c^{2}\right) w+2 a c+a^{2} v-v+2 c$ and $Q_{1}(w)=$ $-(a+1)^{2}+w\left(-1+4 v a+a^{2}-2 a c+2 c\right)$. Clearly, the denominator $Q_{1}^{2}(w)$ is bounded and, thus, $-4 w / Q_{1}^{2}(w)$ is bounded from below be a positive constant. Since $P_{1}(-1)=$ $2 a(c+a v)+2 c(v+a-c+1)>\Theta(a), P_{1}\left(-\frac{a+1}{2 c+1-a}\right)=2(c+1)(a+1)(c+a v) /(2 c+1-a)$ is bounded from below by a positive constant, and $P_{1}$ is linear in $w$, we can conclude that on the whole interval $P_{1}(w)>\Theta(a)$ and, thus, the claim is proved for $i=1$.

Similarly, we can find $\frac{\partial H_{n}^{(2)}}{\partial a}=2 P_{2}(w) / Q_{2}^{2}(w)$, for $w \in\left[-\frac{a+1}{2 c+1-a}, 0\right]$, where $P_{2}(w)=$ $4 a^{2} c(c+1) v^{2} w^{2}+\left(2 c^{2} a^{2}+2 c^{2}+2 a^{4} c-4 c^{3} a^{2}+a^{2}+a^{4}+c-2^{3}+7 a^{2} c-2 c a\right) v w^{2}+c\left(4 a^{3} c+\right.$ $\left.c-6 a^{2} c^{2}-2 c^{2}-3 c a^{2}+1-3 a^{2}+2 a^{3}+6 a c\right) w^{2}-2(c+1)\left(a^{2}-1\right)\left(a^{2}+c\right) v w-2 c(c+1)\left(a^{2}-\right.$ 1) $(2 a-c+1) w+(a+1)^{2}\left(a^{2}+c\right) v+c(a+1)^{2}(2 a-c+1)$, and $Q_{2}(w)=-(a+1)\left(a^{2}-a c+\right.$ $a+c)+\left(3 a^{2} c-a+a^{3}+4 c^{2} a-c\right) w-2 a(a+1) v+2 a(1+c)(a-1) v w$. As the denominator $Q_{2}^{2}(w)$ is bounded, the term $2 / Q_{1}^{2}(w)$ is bounded from below be a positive constant. We also have $P_{2}\left(-\frac{a+1}{2 c+1-a}\right)=4 c a(1+c)(1+a)^{2}(v+a+1-c)(c+a v) /(2 c+1-a)^{2}>\Theta\left(a^{2}\right)$ and $P_{2}(0)=(a+1)^{2}(a(c+a v)+c(v+a+1-c))>\Theta(a)$. Furthermore, since the derivative $\frac{\partial P_{2}(w)}{\partial w}$ is bounded, there exists $\epsilon_{3}>0$ such that, for every $w \in\left[-\epsilon_{3} a^{3 / 2}, 0\right]$, we have $P_{2}(w)>$ $\Theta(a)$. In order to provide a lower bound on $P_{2}(w)$ in the interval $\left[-\frac{a+1}{2 c+1-a},-\epsilon_{3} a^{3 / 2}\right)$, notice first that $P_{2}(w) / w^{2}$ is a quadratic polynomial in $1 / w$, which has minimum at point $1 / w=(1+c)(a-1) /(a+1)$, outside our interval of interest $\left(-\infty,-\frac{2 c+1-a}{a+1}\right]$. Therefore, within this interval, it reaches the global minimum at point $1 / w_{\min }=-\frac{2 c+1-a}{a+1}$. Finally, for $w \in\left[-\frac{a+1}{2 c+1-a},-\epsilon_{3} a^{3 / 2}\right)$, we have $P_{2}(w) \geq \epsilon_{3}^{2} a^{3} P_{2}\left(w_{\text {min }}\right) / w_{\text {min }}^{2} \geq \Theta\left(a^{5}\right)$, since $P_{2}\left(w_{\text {min }}\right)>$ $\Theta\left(a^{2}\right)$.

QED

The rotation number $\rho\left(a_{n}, v_{n}\right)$ of the fractional linear pair $\left(a_{n}, v_{n}\right)$ does not necessarily equal $\rho_{n}=\rho\left(f_{n}, g_{n}\right)$. For that reason, we define the projection operator $\mathcal{P}$ from the space of all commuting pairs $\left(f_{n}, g_{n}\right)$ with well-defined rotation number to $\check{\mathcal{D}}_{c_{n}}$, as $\mathcal{P}\left(f_{n}, g_{n}\right)=$ $\left(a_{n}^{*}, v_{n}^{*}\right)$, where $\left(a_{n}^{*}, v_{n}^{*}\right)=\left(\gamma_{\rho_{n}, c_{n}}\left(v_{n}\right), v_{n}\right)$ if $\left(\gamma_{\rho_{n}, c_{n}}\left(v_{n}\right), v_{n}\right) \in \check{\mathcal{D}}_{c_{n}}$, or let $\left(a_{n}^{*}, v_{n}^{*}\right)$ be the closest to $\left(a_{n}, v_{n}\right)$ intersection point of the curve $a=\gamma_{\rho_{n}, c_{n}}(v)$ with the boundary of $\check{\mathcal{D}}_{c_{n}}$. Since this projection is determined uniquely by $f_{n}$, and the rotation number $\rho_{n}$, and since we will always have in mind the $n$-th renormalizations of a particular circle map $T$, we will write $\mathcal{P} f_{n}=\left(a_{n}^{*}, v_{n}^{*}\right)$.

Proposition 4.8 Let $\lambda_{1^{-}}>\sqrt[6]{\lambda}$. Then, $\left|\gamma_{\rho_{n}, c_{n}}\left(v_{n}\right)-a_{n}\right|<\Theta\left(\left(\lambda / \lambda_{1^{-}}^{6}\right)^{n}\right)$, for sufficiently large $n$ in the initial string and for all $n \in\left[n_{1}+1, n_{2}-1\right]$ in an ordinary string.

Proof. We assume that $a_{n}>\gamma_{\rho_{n}, c_{n}}\left(v_{n}\right)$. In the opposite case, the proof is similar and even simpler. It follows from Proposition 4.6 that, for all $w \in[-1,0]$, we have

$$
H_{f_{n}, g_{n}}(w) \geq H_{n}\left(w ; a_{n}, v_{n}\right)-\frac{C_{2}}{a_{n}} \lambda^{n} .
$$


Since $\left(a_{n}, v_{n}\right) \in \Phi_{c_{n}}^{\varepsilon}$, for large enough $n$ in the initial string and for $n_{1}<n<n_{2}$ in an ordinary string, at least half of the segment $\left[\gamma_{\rho_{n}, c_{n}}\left(v_{n}\right), a_{n}\right] \times\left\{v_{n}\right\}$ lies inside $\Phi_{c_{n}}^{\varepsilon / 2}$. Moreover, that is the segment $\left[\hat{a}_{n}, a_{n}\right] \times\left\{v_{n}\right\}$, for some $\hat{a}_{n} \in\left[\gamma_{\rho_{n}, c_{n}}\left(v_{n}\right), a_{n}\right]$. To see this, notice that $\left(\gamma_{\rho_{n}, c_{n}}\left(v_{n}\right), v_{n}\right)$ belongs to $\Phi_{c_{n}}^{0}$ and that the distance between the bottom boundaries of the regions $\Phi_{c_{n}}^{\varepsilon}$ and $\Phi_{c_{n}}^{0} \supset \Phi_{c_{n}}^{\varepsilon}$, in the direction of coordinate $a$, is proportional to $\varepsilon$.

Using Proposition 4.7, we find

$$
H_{f_{n}, g_{n}}(w) \geq H_{n}\left(w ; \gamma_{\rho_{n}, c_{n}}\left(v_{n}\right), v_{n}\right)+\mu_{c_{n}, \varepsilon / 2} \frac{a_{n}^{6}-\hat{a}_{n}^{6}}{6}-\frac{C_{2}}{a_{n}} \lambda^{n} .
$$

If $\mu_{c_{n}, \varepsilon / 2} \frac{a_{n}^{6}-\hat{a}_{n}^{6}}{6}-\frac{C_{2}}{a_{n}} \lambda^{n}>0$, then $H_{f_{n}, g_{n}}(w)>H_{n}\left(w ; \gamma_{\rho_{n}, c_{n}}\left(v_{n}\right), v_{n}\right)$ for all $w \in[-1,0]$. This gives that the rotation number $\rho\left(f_{n}, g_{n}\right)>\rho\left(\gamma_{\rho_{n}, c_{n}}\left(v_{n}\right), v_{n}\right)$, while, in fact, they are equal. Thus,

$$
a_{n}^{6}-\hat{a}_{n}^{6} \leq \frac{6 C_{2} \lambda^{n}}{a_{n} \mu_{c_{n}, \varepsilon / 2}} \leq \frac{6 C_{2} \lambda^{n}}{\sigma \lambda_{1^{-}}^{n} \min \left\{\mu_{c, \varepsilon / 2}, \mu_{c^{-1}, \varepsilon / 2}\right\}} .
$$

The claim follows since $a_{n}-\gamma_{\rho_{n}, c_{n}}\left(v_{n}\right) \leq 2\left(a_{n}-\hat{a}_{n}\right) \leq 2\left(a_{n}^{6}-\hat{a}_{n}^{6}\right) / a_{n}^{5}$.

Lemma 4.9 If $\lambda_{1^{-}}>\sqrt[6]{\lambda}$, then $a_{n}^{*}-a_{n}=\mathcal{O}\left(\left(\lambda / \lambda_{1^{-}}^{6}\right)^{n}\right)$ and $v_{n}^{*}-v_{n}=\mathcal{O}\left(\left(\lambda / \lambda_{1^{-}}^{6}\right)^{n}\right)$, for sufficiently large $n$ in the initial string and for all $n \in\left[n_{1}+1, n_{2}-1\right]$ in an ordinary string.

Proof. The claim follows from the definition of the projection operator $\mathcal{P}$, Proposition 4.3 and Proposition 4.8. Just notice that the slope of the curves $a=\frac{c_{n}\left(c_{n}-v-1\right)}{v}$ and $a=\frac{4 c_{n}\left(v+1-c_{n}\right)}{\left(c_{n}-1\right)^{2}}$ inside of $\check{\mathcal{D}}_{c_{n}}$ are bounded away from the interval $(-1,0)$ and thus their intersection with $\gamma_{\rho_{n}, c_{n}}$ is transversal. $\quad$ QED

On the set of commuting pairs $(F, G)$ in $\Phi_{c_{n}}^{\varepsilon}$, we consider two sets of coordinates $(a, v)$ and $(x, y)$, where

$$
(x, y)=\mathcal{U}(a, v)=\left(a v, \frac{v+1-c}{c a}\right) .
$$

The coordinates $x$ and $y$ can be viewed as independent indicators of nonlinearity of $F$ and $G$. To see this, notice that if we perform a linear scaling $z=a t$, the map $F_{a, v, c}(z)$ is transformed into $F_{c}(t)=\frac{1+c t}{1-x t}$. Similarly, by simple inversion $z=-t, G_{a, v, c}(z)$ is transformed into $G_{c}(t)=\frac{1+t / c}{1-y t}$. The following corollary follows directly from Lemma 4.9.

Corollary 4.10 If $\lambda_{1^{-}}>\sqrt[8]{\lambda}$, then $y_{n}^{*}-y_{n}=\mathcal{O}\left(\left(\lambda / \lambda_{1^{-}}^{8}\right)^{n}\right)$, for sufficiently large $n$ in the initial string and for all $n \in\left[n_{1}+1, n_{2}-1\right]$ in an ordinary string.

Proposition $4.11([18])$ For any $(x, y) \in \check{\mathcal{D}}_{c}, \mathcal{R}_{c}(x, y)=\left(x^{\prime}, y^{\prime}\right)$ with $y^{\prime}=x$. 


\section{Renormalizations with small $a_{n}$}

In this section we consider the case when $c_{n}<1$, since in the opposite case $a_{n}$ is bounded from below by a positive constant due to Proposition 3.2.

Lemma 5.1 Let $\lambda_{2} \in\left(\lambda_{1^{+}}, 1\right)$. For sufficiently small $\sigma>0$ and sufficiently large $n \in \mathbb{N}$, if $a_{n} \leq \sigma \lambda_{1^{+}}^{n}$, then $a_{n}^{*}-a_{n}=\mathcal{O}\left(\lambda_{2}^{n}\right)$ and $v_{n}^{*}-v_{n}=\mathcal{O}\left(\lambda_{2}^{n}\right)$. Also, $\mathcal{P} f_{n}=\left(a_{n}^{*}, v_{n}^{*}\right)$ and $\mathcal{R}_{c_{n-1}} \mathcal{P} f_{n-1}=\left(\bar{a}_{n}, \bar{v}_{n}\right)$ are $\mathcal{O}\left(\lambda_{2}^{n}\right)$-close, as points in $\check{\mathcal{D}}_{c_{n}}$.

Proof. It follows from [14] (see Proposition 3.2 and Proposition 3.4 therein) that for every $\varkappa>0$ there exists a constant $C_{3}>0$ such that for sufficiently large $n \in \mathbb{N}$ and sufficiently large $k_{n+1}(\sigma>0$ sufficiently small $)$, we have $\gamma_{1}^{-(\chi+\varkappa) k_{n+1}} \leq C_{3} a_{n} \leq C_{3} \sigma \lambda_{1+}^{n}$, where $\gamma_{1}=\left(f_{n}\right)_{+}^{\prime}(-1), \gamma_{2}=\left(f_{n}\right)_{-}^{\prime}(0), \chi=\frac{\ln \gamma_{2}}{\ln \gamma_{2}+\ln \gamma_{1}^{-1}}$ and, thus, $k_{n+1}>\frac{n \ln \lambda_{1+}^{-1}-\ln \left(C_{3} \sigma\right)}{(\chi+\varkappa) \ln \gamma_{1}}$. Since the map $f_{n}$ is exponentially close to a fractional linear $F_{a_{n}, v_{n}, c_{n}}$, in the $C^{2}$-topology, due to (B), and $v_{n}=c_{n}-1+\mathcal{O}\left(a_{n}\right)$ is, due to our assumption on $a_{n}$, exponentially close to $c_{n}-1$, it follows that $\gamma_{1}$ and $\gamma_{2}$ are exponentially close to $c_{n}^{-1}$ and $c_{n}$, respectively. Consider now an arbitrary fractional linear map $F_{a_{n}^{\prime}, v_{n}^{\prime}, c_{n}}$, with the same height $k_{n+1}$ as $f_{n}$, and with corresponding derivatives $\gamma_{1}^{\prime}$ and $\gamma_{2}^{\prime}$, at -1 and 0 , respectively. Let $\chi^{\prime}=\frac{\ln \gamma_{2}^{\prime}}{\ln \gamma_{2}^{\prime}+\ln \left(\gamma_{1}^{\prime}\right)^{-1}}$. Using once more Proposition 3.2 and Proposition 3.4 of [14], we find that for every $\varkappa^{\prime}>0$ and $\sigma>0$ sufficiently small

$$
a_{n}^{\prime} \leq C_{4}\left(\gamma_{1}^{\prime}\right)^{-\left(\chi^{\prime}-\varkappa^{\prime}\right) k_{n+1}} \leq C_{4}\left(\gamma_{1}^{\prime}\right)^{-\frac{1}{\ln \gamma_{1}}} \frac{\chi^{\prime}-\varkappa^{\prime}}{\chi+\varkappa}\left(n \ln \lambda_{1+}^{-1}-\ln \left(C_{3} \sigma\right)\right)=C_{4} e^{\frac{\ln \gamma_{1}^{\prime}}{\ln \gamma_{1}} \frac{\chi^{\prime}-\varkappa^{\prime}}{\chi+\varkappa} \ln \left(C_{3} \sigma \lambda_{1+}^{n}\right)},
$$

for some $C_{4}>0$. By choosing $\sigma>0$ small enough, we can make $\gamma_{1}^{\prime}$ and $\gamma_{2}^{\prime}$ arbitrarily close to $c_{n}^{-1}$ and $c_{n}$, respectively, and thus $\frac{\ln \gamma_{1}^{\prime}}{\ln \gamma_{1}} \frac{\chi^{\prime}-\varkappa^{\prime}}{\chi+\varkappa}$ can be made arbitrarily close to 1 . Therefore, for every $\lambda_{2}>\lambda_{1^{+}}$, there exists $C_{5}>0$, such that

$$
a_{n}^{\prime} \leq C_{4}\left(C_{3} \sigma \lambda_{1+}^{n}\right)^{\frac{\ln \gamma_{1}^{\prime}}{\ln \gamma_{1}} \frac{\chi^{\prime}-\varkappa^{\prime}}{\chi+\varkappa}} \leq C_{5} \lambda_{2}^{n}
$$

In particular, this is true for $a_{n}^{\prime}=a_{n}^{*}$, i.e. the first component of $\mathcal{P} f_{n}$, and the first component of $\mathcal{R}_{c_{n-1}} \mathcal{P} f_{n-1}$. The estimates on the second components follow from the fact that these two points belong to $\check{\mathcal{D}}_{c_{n}}$.

QED

\section{Renormalization and projection operators}

On the set of commuting pairs in $\Phi_{c}^{\varepsilon} \cup \check{\mathcal{D}}_{c}$, we consider two metrics: the standard metric

$$
d((a, v),(\widetilde{a}, \widetilde{v}))=|a-\widetilde{a}|+|v-\widetilde{v}| .
$$


and the metric

$$
d_{c}((x, y),(\widetilde{x}, \widetilde{y}))=|x-\widetilde{x}|+|y-\widetilde{y}| .
$$

Here, the parameters $(a, v)$ and $(x, y)$ correspond to a pair $(F, G)$, and $(\widetilde{a}, \widetilde{v})$ and $(\widetilde{x}, \widetilde{y})$ correspond to $(\widetilde{F}, \widetilde{G})$.

Proposition 6.1 Let $\lambda_{1^{-}}>\sqrt[8]{\lambda}$ and $\lambda_{2}>\lambda / \lambda_{1^{-}}^{8}$. For sufficiently large $n$ in the initial string, and for all $n$ in an ordinary string, such that $n_{1} \leq n<n_{2}-1$, we have $d_{c_{n+1}}\left(\mathcal{P} f_{n+1}, \mathcal{R}_{c_{n}} \mathcal{P} f_{n}\right)=\mathcal{O}\left(\lambda_{2}^{n}\right)$.

Proof. Let $\mathcal{R}_{c_{n}}\left(a_{n}^{*}, v_{n}^{*}\right)=\left(\bar{a}_{n+1}, \bar{v}_{n+1}\right)$. It follows directly from (4.1) that $y_{n+1}=x_{n}+$ $\mathcal{O}\left(\left(\lambda / \lambda_{1^{-}}\right)^{n}\right)$, for sufficiently large $n$ in the initial string and for $n_{1} \leq n<n_{2}-1$ in an ordinary string. It follows from Proposition 4.11 and Lemma 4.9 that $\bar{y}_{n+1}=x_{n}^{*}=$ $x_{n}+\mathcal{O}\left(\left(\lambda / \lambda_{1^{-}}^{6}\right)^{n}\right)$, for sufficiently large $n$ in the initial string and for $n_{1}<n \leq n_{2}-1$ in an ordinary string. Lemma 5.1 gives us $\bar{y}_{n_{1}+1}=x_{n_{1}}^{*}=x_{n_{1}}+\mathcal{O}\left(\lambda_{2}^{n_{1}}\right)$. Therefore, we obtain $\bar{y}_{n+1}-y_{n+1}=\mathcal{O}\left(\lambda_{2}^{n}\right)$, for sufficiently large $n$ in the initial string and for all $n$ such that $n_{1} \leq n<n_{2}-1$ in an ordinary string. Using Corollary 4.10, we find $\bar{y}_{n+1}-y_{n+1}^{*}=\mathcal{O}\left(\lambda_{2}^{n}\right)$, also for sufficiently large $n$ in the initial string and for all $n$ such that $n_{1} \leq n<n_{2}-1$ in an ordinary string.

Recall that the slope of the curve $\gamma_{\rho_{n+1}, c_{n+1}}$ lies in the interval $(-1,0)$. On the other hand, the slopes of the curves $y=\bar{y}_{n+1}$ and $y=y_{n+1}^{*}$, in the $(v, a)$ plane, lie outside of this interval. In what follows, we will show this claim for the first of these curves only, as the same argument applies to the second curve. Notice first that these curves are straight lines $a=\frac{v+1-c_{n+1}}{c_{n+1} \bar{y}_{n+1}}$ and $a=\frac{v+1-c_{n+1}}{c_{n+1} y_{n+1}^{*}}$ with slopes $\frac{d a}{d v}=\left(c_{n+1} \bar{y}_{n+1}\right)^{-1}$ and $\frac{d a}{d v}=\left(c_{n+1} y_{n+1}^{*}\right)^{-1}$, respectively. Since $\left(\bar{a}_{n+1}, \bar{v}_{n+1}\right) \in \check{\mathcal{D}}_{c_{n+1}}$, it follows directly from the definition of $\check{\mathcal{D}}_{c_{n+1}}$ that

$$
\left|\frac{d a}{d v}\right|=\left|\frac{\bar{a}_{n+1}}{\bar{v}_{n+1}+1-c_{n+1}}\right| \geq\left|\frac{c_{n+1}}{\bar{v}_{n+1}}\right| \geq \frac{1}{\left|c_{n}-1\right|} .
$$

If $c_{n}>1$, then $\frac{d a}{d v}>0$ and, thus, the slope of the line $y=\bar{y}_{n+1}$ lies in the interval $\left[\frac{1}{c_{n}-1}, \infty\right)$ (in fact, it is in $\left[\frac{1}{c_{n}-1}, \frac{4 c}{\left(c_{n}-1\right)^{2}}\right)$ ). If $c_{n}<1$, then $\frac{d a}{d v}<0$ and, thus, the slope of this line belongs to the interval $\left(-\infty, \frac{1}{c_{n}-1}\right]$. In both cases, the slope of the line $y=\bar{y}_{n+1}$ is bounded away from the interval $(-1,0)$. The same can be said about the line $y=y_{n+1}^{*}$. Hence, the intersections of these lines with the curve $\gamma_{\rho_{n+1}, c_{n+1}}$ are uniformly transversal, i.e. the (positive) angles between these lines and the curve, are greater than some positive constant. Therefore, the distance of the intersection points of these lines with the curve $\gamma_{\rho_{n+1}, c_{n+1}}$, i.e. $d\left(\left(\bar{a}_{n+1}, \bar{v}_{n+1}\right),\left(a_{n+1}^{*}, v_{n+1}^{*}\right)\right)$, is bounded from above by the (positive) angle between these lines, and, thus, $d\left(\left(\bar{a}_{n+1}, \bar{v}_{n+1}\right),\left(a_{n+1}^{*}, v_{n+1}^{*}\right)\right)<\Theta\left(\left|\bar{y}_{n+1}-y_{n+1}^{*}\right|\right)$. The latter estimate follows from the formula $\tan \bar{\theta}-\tan \theta^{*}=\frac{\sin \left(\bar{\theta}-\theta^{*}\right)}{\cos \bar{\theta} \cos \theta^{*}}$, where $\bar{\theta}$ and $\theta^{*}$ are the angles between the $v$ axis the lines perpendicular to $y=\bar{y}_{n+1}$ and $y=y_{n+1}^{*}$, respectively. Finally, for sufficiently large $n$ in the initial string and for all $n$ such that $n_{1} \leq n<n_{2}-1$ in an ordinary string, we obtain $\bar{x}_{n+1}-x_{n+1}^{*}=\mathcal{O}\left(\lambda_{2}^{n}\right)$. 


\section{Convergence of renormalizations}

On the set of renormalizable commuting pairs in $\check{\mathcal{D}}_{c}$, with the same irrational rotation number, the renormalization operator is Lipschitz and the two-step renormalization operator is a contraction, in the metric $d_{c}$.

Proposition 7.1 ([18]) For every positive $c \neq 1$, there exist constants $B$ and $\beta \in(0,1)$ such that for any two points $(a, v)$ and $(\widetilde{a}, \widetilde{v})$ in $\check{\mathcal{D}}_{c} \backslash\{(0, c-1)\}$, with the same irrational rotation number, and corresponding coordinates $(x, y)$ and $(\widetilde{x}, \widetilde{y})$, respectively, we have

$$
d_{\frac{1}{c}}\left(\mathcal{R}_{c}(\widetilde{x}, \widetilde{y}), \mathcal{R}_{c}(x, y)\right) \leq B d_{c}((\widetilde{x}, \widetilde{y}),(x, y))
$$

and

$$
d_{c}\left(\mathcal{R}_{\frac{1}{c}} \circ \mathcal{R}_{c}(\widetilde{x}, \widetilde{y}), \mathcal{R}_{\frac{1}{c}} \circ \mathcal{R}_{c}(x, y)\right) \leq \beta d_{c}((\widetilde{x}, \widetilde{y}),(x, y)) .
$$

On the other hand, the $C^{2}$-norm of the distance of fractional linear maps is easily controlled by their distance in the $d$ metric.

Proposition 7.2 There exists $C_{6}>0$ such that, if $\min \{\widetilde{v}, v\}>-1+\epsilon_{4}$ for some $\epsilon_{4}>0$, we have

$$
\left\|F_{\widetilde{a}, \widetilde{v}, c}-F_{a, v, c}\right\|_{C^{2}} \leq C_{6}(|\widetilde{a}-a|+|\widetilde{v}-v|)
$$

Proof. Since

$$
F_{\widetilde{a}, \tilde{v}, c}-F_{a, v, c}=\frac{\widetilde{a}-a}{1-\widetilde{v} z}+\frac{(a+c z)(\widetilde{v}-v) z}{(1-v z)(1-\widetilde{v} z)},
$$

$(1-v z)$ and $(1-\widetilde{v} z)$ are bounded away from zero on $[-1,0]$, and all other variables are bounded, the claim follows.

QED

The following proposition provides a relation between the metrics.

Proposition 7.3 There exists $K>1$, such that for any two commuting pairs $(a, v)$ and $(\widetilde{a}, \widetilde{v})$ in $\check{\mathcal{D}}_{c} \backslash\{(0, c-1)\}$, on the same curve $\gamma_{\rho, c}$, and with corresponding coordinates $(x, y)$ and $(\widetilde{x}, \widetilde{y})$, we have

$$
K^{-1} d((\widetilde{a}, \widetilde{v}),(a, v)) \leq d_{c}((\widetilde{x}, \widetilde{y}),(x, y)) \leq \frac{K}{a \widetilde{a}} d((\widetilde{a}, \widetilde{v}),(a, v))
$$

Proof. The first inequality follows from the second of the inverse relations

$$
a=\frac{c-1}{2 c y}\left(-1+\sqrt{1+\frac{4 c x y}{(c-1)^{2}}}\right), \quad v=\frac{c-1}{2}\left(1+\sqrt{1+\frac{4 c x y}{(c-1)^{2}}}\right) .
$$

The only situation when we use the fact that both $(a, v)$ and $(\widetilde{a}, \widetilde{v})$ belong to the same curve $\gamma_{\rho, c}$ is when $\frac{4 c x y}{(c-1)^{2}}$ is close to -1 . In $\check{\mathcal{D}}_{c}$, this is only the case when $a$ is close to $c$, 
$v$ is close to $\frac{c-1}{2}$ and, thus, $y$ is close to $\frac{1-c}{2 c^{2}}$. The level sets of $y$ in the $(v, a)$ plane are straight lines with slope $\frac{1}{c y}$, which is close to $\frac{2 c}{1-c}$. These slopes are bounded away from the interval $(-1,0)$ and, thus, the intersection with $\gamma_{\rho, c}(v)$ is transversal. Therefore, $|a-\widetilde{a}|$ and $|v-\widetilde{v}|$ (and, thus, $|x-\widetilde{x}|$ ) are of the same order as $|y-\widetilde{y}|$. The second inequality follows from direct relations (4.24).

QED

Remark 1 Without the assumption that the pairs belong to the same curve $\gamma_{\rho, c}$ in Proposition 7.3 , one would have a weaker inequality $d^{2}((\widetilde{a}, \widetilde{v}),(a, v)) \leq K d_{c}((\widetilde{x}, \widetilde{y}),(x, y))$.

Proposition 7.4 Let $\widetilde{\lambda}_{1^{-}}<\lambda_{1}<\widetilde{\lambda}_{1^{+}}$. Consider the sequences of renormalizations $\left(f_{n}, g_{n}\right)$ and $\left(\widetilde{f}_{n}, \widetilde{g}_{n}\right), n \in \mathbb{N}_{0}$, of any two circle maps $T$ and $\widetilde{T}$ with a break of size $c$ and the same irrational rotation number $\rho$, with corresponding parameters $\left(a_{n}, v_{n}\right)$ and $\left(\widetilde{a}_{n}, \widetilde{v}_{n}\right)$, respectively. There exist $C_{7}, C_{8}>0$ such that for sufficiently large $n \in \mathbb{N}$, if $a_{n} \leq \sigma \lambda_{1}^{n}$, for some $\sigma>0$ sufficiently small then $\widetilde{a}_{n} \leq C_{7} \sigma \widetilde{\lambda}_{1^{+}}^{n}$. If $a_{n}>\sigma \lambda_{1}^{n}$, then $\widetilde{a}_{n}>C_{8} \sigma \widetilde{\lambda}_{1^{-}}^{n}$.

Proof. The proof of the first claim is similar to the proof of Lemma 5.1. The proof of the second claim is by contrapositive.

QED

Remark 2 Proposition 7.4 allows us to partition the two sequences of renormalizations for two maps $T$ and $\widetilde{T}$, with the same irrational rotation number, into finite or infinite sequence strings $S_{i}=\left\{f_{n_{1}(i)}, \ldots, f_{n_{2}(i)-1}\right\}$ and $\widetilde{S}_{i}=\left\{\widetilde{f}_{n_{1}(i)}, \ldots, \widetilde{f}_{n_{2}(i)-1}\right\}$, with $1 \leq i<N$, $N \in \mathbb{N} \cup\{\infty\}$ and $n_{2}(i)=n_{1}(i+1)$, in such a way that, for each $i$, the lengths of the $i$-th strings are the same. More precisely, starting with some $n_{0} \in \mathbb{N}$, we can partition the sequence of renormalizations for $T$ indexed by $n \geq n_{0}$ into strings $S_{i}$ of lengths $n_{2}(i)-n_{1}(i)$ uniquely, by choosing $\sigma>0$ and $\lambda_{1^{-}}=\lambda_{1^{+}}=\lambda_{1} \in(\lambda, 1)$. The sequence of renormalizations for $\widetilde{T}$ can then be partitioned into strings $\widetilde{S}_{i}$ of the same lengths $n_{2}(i)-n_{1}(i)$, with some $\widetilde{\sigma}=\Theta(\sigma), \widetilde{\lambda}_{1^{-}} \in\left(\sqrt[8]{\lambda}, \lambda_{1}\right)$ and $\widetilde{\lambda}_{1^{+}} \in\left(\lambda_{1}, \lambda_{2}\right)$.

Lemma 7.5 There exists $C_{9}>0$ such that, for $\lambda_{3} \in\left(\max \left\{\beta^{1 / 2}, \lambda_{2}\right\}, 1\right)$ and for sufficiently large $n$ in the initial string and for every $n \in\left[n_{1}+1, n_{2}-1\right]$ in an ordinary string, we have

$$
d_{c_{n}}\left(\mathcal{P} \widetilde{f}_{n}, \mathcal{P} f_{n}\right) \leq C_{9} \lambda_{3}^{n} .
$$

Proof. Using the triangle inequality, we find

$$
\begin{array}{r}
d_{c_{n}}\left(\mathcal{P} \tilde{f}_{n}, \mathcal{P} f_{n}\right) \leq d_{c_{n}}\left(\mathcal{P} \widetilde{f}_{n}, \mathcal{R}_{c_{n-1}} \mathcal{P} \widetilde{f}_{n-1}\right)+d_{c_{n}}\left(\mathcal{P} f_{n}, \mathcal{R}_{c_{n-1}} \mathcal{P} f_{n-1}\right) \\
+d_{c_{n}}\left(\mathcal{R}_{c_{n-1}} \mathcal{P} \widetilde{f}_{n-1}, \mathcal{R}_{c_{n-1}} \mathcal{P} f_{n-1}\right) .
\end{array}
$$

It follows from Proposition 6.1 that, for sufficiently large $n$ in the initial string and for every $n_{1}<n<n_{2}$ in an ordinary string, we have $d_{c_{n}}\left(\mathcal{P} f_{n}, \mathcal{R}_{c_{n-1}} \mathcal{P} f_{n-1}\right) \leq C_{10} \lambda_{2}^{n}$ 
and $d_{c_{n}}\left(\mathcal{P} \widetilde{f}_{n}, \mathcal{R}_{c_{n-1}} \mathcal{P} \widetilde{f}_{n-1}\right) \leq C_{10} \lambda_{2}^{n}$, for some $C_{10}>0$, assuming that we have chosen $\widetilde{\lambda}_{1-}>\sqrt[8]{\lambda}$ and $\widetilde{\lambda}_{1+}<\lambda_{2}$. Applying (7.8) recursively, in a string of more than two renormalizations, and using Proposition 7.1, we obtain

$$
\begin{array}{r}
d_{c_{n}}\left(\mathcal{P} \tilde{f}_{n}, \mathcal{P} f_{n}\right) \leq 2 C_{10} \lambda_{2}^{n}+d_{c_{n}}\left(\mathcal{R}_{c_{n-1}} \circ \mathcal{R}_{c_{n-2}} \mathcal{P} \tilde{f}_{n-2}, \mathcal{R}_{c_{n-1}} \circ \mathcal{R}_{c_{n-2}} \mathcal{P} f_{n-2}\right) \\
+d_{c_{n}}\left(\mathcal{R}_{c_{n-1}} \mathcal{P} \widetilde{f}_{n-1}, \mathcal{R}_{c_{n-1}} \circ \mathcal{R}_{c_{n-2}} \mathcal{P} \widetilde{f}_{n-2}\right)+d_{c_{n}}\left(\mathcal{R}_{c_{n-1}} \mathcal{P} f_{n-1}, \mathcal{R}_{c_{n-1}} \circ \mathcal{R}_{c_{n-2}} \mathcal{P} f_{n-2}\right) \\
\leq 2\left(1+B \lambda_{2}^{-1}\right) C_{10} \lambda_{2}^{n}+d_{c_{n}}\left(\mathcal{R}_{c_{n-1}} \circ \mathcal{R}_{c_{n-2}} \mathcal{P} \tilde{f}_{n-2}, \mathcal{R}_{c_{n-1}} \circ \mathcal{R}_{c_{n-2}} \mathcal{P} f_{n-2}\right) \\
\leq 2\left(1+B \lambda_{2}^{-1}\right) C_{10} \lambda_{2}^{n}+\beta d_{c_{n}}\left(\mathcal{P} \widetilde{f}_{n-2}, \mathcal{P} f_{n-2}\right)
\end{array}
$$

By iterating the resulting inequality, we obtain

$$
\begin{array}{r}
d_{c_{n}}\left(\mathcal{P} \tilde{f}_{n}, \mathcal{P} f_{n}\right) \leq 2\left(1+B \lambda_{2}^{-1}\right) C_{10} \sum_{i=0}^{k-1} \lambda_{2}^{n-2 i} \beta^{i}+\beta^{k} d_{c_{n}}\left(\mathcal{P} \tilde{f}_{n-2 k}, \mathcal{P} f_{n-2 k}\right) \\
\leq C_{11} \lambda_{4}^{n}+\beta^{k} d_{c_{n}}\left(\mathcal{P} \tilde{f}_{n-2 k}, \mathcal{P} f_{n-2 k}\right)
\end{array}
$$

for some $\lambda_{4}>\max \left\{\beta^{1 / 2}, \lambda_{2}\right\}$ and $C_{11}>0$. If $d_{c_{n}}\left(\mathcal{P} \widetilde{f}_{n-2 k}, \mathcal{P} f_{n-2 k}\right) \leq C_{9} \lambda_{3}^{n-2 k}, n_{1}<$ $n-2 k<n<n_{2}$, for some $\lambda_{3}>\lambda_{4}$ and $C_{9}>0$, then $d_{c_{n}}\left(\mathcal{P} \tilde{f}_{n}, \mathcal{P} f_{n}\right) \leq \lambda_{3}^{n}\left(C_{11}\left(\lambda_{4} / \lambda_{3}\right)^{n}+\right.$ $\left.C_{9}\left(\sqrt{\beta} / \lambda_{3}\right)^{2 k}\right) \leq C_{9} \lambda_{3}^{n}$, if $C_{9}$ is large enough.

To complete the proof by induction, we need to verify that the estimates are also true for $n=n_{1}+1$ and $n=n_{1}+2$, in an ordinary string. In the initial string, the initial estimates are certainly satisfied, for some large $n_{1}^{\prime}$ and $n_{1}^{\prime}+1$, if $C_{9}$ is chosen sufficiently large. For an ordinary string and $n=n_{1}+1$ (if smaller than $n_{2}$ ), we have from (7.8) and Proposition 6.1 that $d_{c_{n}}\left(\mathcal{P} \widetilde{f}_{n}, \mathcal{P} f_{n}\right) \leq 2 C_{10} \lambda_{2}^{n}+d_{c_{n}}\left(\mathcal{R}_{c_{n-1}} \mathcal{P} \widetilde{f}_{n-1}, \mathcal{R}_{c_{n-1}} \mathcal{P} f_{n-1}\right)$. Using similar reasoning as in the proof of Proposition 6.1, we find $d_{c_{n}}\left(\mathcal{R}_{c_{n-1}} \mathcal{P} \widetilde{f}_{n-1}, \mathcal{R}_{c_{n-1}} \mathcal{P} f_{n-1}\right) \leq$ $C_{12}\left|\bar{y}_{n}-\bar{y}_{n}\right|=C_{12}\left|\widetilde{x}_{n-1}^{*}-x_{n-1}^{*}\right| \leq C_{13} \lambda_{2}^{n}$, for some $C_{12}, C_{13}>0$. The equality follows from Proposition 4.11. In the last inequality, we have used Lemma 5.1 and Proposition 7.4. For $n=n_{1}+2$ (if smaller than $n_{2}$ ), the claim follows from (7.8), using the estimate on $d_{c_{n_{1}+1}}\left(\mathcal{P} \widetilde{f}_{n_{1}+1}, \mathcal{P} f_{n_{1}+1}\right)$, Proposition 6.1 and Proposition 7.1.

QED

Proof of Theorem 1.1. Using the triangle inequality and Proposition 7.2, we find

$$
\left\|\tilde{f}_{n}-f_{n}\right\|_{C^{2}} \leq\left\|\tilde{f}_{n}-\mathcal{P} \tilde{f}_{n}\right\|_{C^{2}}+\left\|f_{n}-\mathcal{P} f_{n}\right\|_{C^{2}}+C_{6} d\left(\mathcal{P} \tilde{f}_{n}, \mathcal{P} f_{n}\right)
$$

For $n$ sufficiently large belonging to the initial string and $n_{1}<n<n_{2}$ belonging to an ordinary string, we have $\left\|f_{n}-\mathcal{P} f_{n}\right\|_{C^{2}} \leq C_{14}\left(\lambda / \lambda_{1}^{6}\right)^{n}$, for some $C_{14}>0$, as follows from property (B) and Lemma 4.9. Therefore, for some $C_{15}>0$, we have

$$
\left\|\tilde{f}_{n}-f_{n}\right\|_{C^{2}} \leq C_{15}\left(\lambda / \lambda_{1}^{6}\right)^{n}+C_{15}\left(\lambda / \widetilde{\lambda}_{1^{-}}^{6}\right)^{n}+K C_{6} d_{c_{n}}\left(\mathcal{P} \tilde{f}_{n}, \mathcal{P} f_{n}\right)
$$


Using Lemma 7.5, we obtain, from this estimate, for some $C_{16}>0$,

$$
\left\|\widetilde{f}_{n}-f_{n}\right\|_{C^{2}} \leq C_{16} \lambda_{3}^{n}
$$

It remains to prove the same estimate for $n=n_{1}$ in every ordinary sting. For such $n$, this estimate follows directly from

$$
\left\|\widetilde{f}_{n}-f_{n}\right\|_{C^{2}} \leq\left\|\widetilde{f}_{n}-\widetilde{F}_{n}\right\|_{C^{2}}+\left\|f_{n}-F_{n}\right\|_{C^{2}}+C_{6} d\left(\left(\widetilde{a}_{n}, \widetilde{v}_{n}\right),\left(a_{n}, v_{n}\right)\right),
$$

using property (B), Proposition 7.4 and Proposition 3.4.

It follows from Proposition 3.5 and the estimates above that the constant $\mu=\lambda_{3}$ can be chosen uniformly. It depends only on the size of the break, $c$, and does not depend on the rotation number of the maps.

QED

\section{References}

[1] G. Arioli, H. Koch, The critical renormalization fixed point for commuting pairs of area-preserving maps, Comm. Math. Phys. 2952 (2010), 415-429.

[2] V. I. Arnol'd, Small denominators I: On the mapping of a circle into itself, Izv. Akad. Nauk. Math. Serie 25 (1961), 21-86; Transl. A.M.S. Serie 246 (1965).

[3] A. Avila, R. Krikorian, Reducibility or nonuniform hyperbolicity of quasiperiodic Schrödinger cocycles, Ann. Math. 1644 (2006), 911-940.

[4] K. Chunha and D. Smania, Renormalization for piecewise smooth homeomorphisms on the circle Preprint: arXiv 1108.1968 (2011).

[5] K. Chunha and D. Smania, Rigidity for piecewise smooth homeomorphisms on the circle Preprint: arXiv 1201.1401 (2012).

[6] P. Coullet and C. Tresser, Iteration d'endomorphismes et groupe de renormalisation, J. Phys. Colloque 39, (1978) C5-25.

[7] M. J. Feigenbaum, Quantitative universality for a class of nonlinear transformations, J. Stat. Phys. 19 (1978), 25-52.

[8] M. J. Feigenbaum, The universal metric properties of nonlinear transformations, $J$. Stat. Phys. 21 (1979), 669.

[9] E. de Faria, W. de Melo, Rigidity of critical circle maps I, J. Eur. Math. Soc. 14 (1999), 339-392. 
[10] E. de Faria, W. de Melo, Rigidity of critical circle maps II, J. Am. Math. Soc. 132 (2000), 343-370.

[11] M. R. Herman, Sur la conjugasion differentiable des difféomorphismes du cercle a de rotations, Publ. Math. Inst. Hautes Etudes Sci. 49 (1979), 5-234.

[12] Y. Katznelson, D. Orstein, The differentiability of conjugation of certain diffeomorphisms of the circle, Ergod. Th. E Dynam. Sys. 9 (1989), 643-680.

[13] K. Khanin, D. Khmelev, Renormalizations and rigidity theory for circle homeomorphisms with singularities of break type, Commun. Math. Phys. 2351 (2003), 69-124.

[14] K. Khanin, S. Kocić, Abscence of robust rigidity for circle diffeomorphisms with breaks, Ann. Inst. H. Poincaré Anal. Non Linéaire, to appear (2012).

[15] K. Khanin, S. Kocić, E. Mazzeo, $C^{1}$-rigidity of circle diffeomorphisms with breaks for almost all rotation numbers, submitted (2011).

[16] K. Khanin, A. Teplinsky, Robust rigidity for circle diffeomorphisms with singularities, Invent. Math. 169 (2007), 193-218.

[17] K. Khanin, A. Teplinsky, Herman's theory revisited, Invent. Math. 178 (2009), 333344.

[18] K. Khanin, A. Teplinsky, Renormalization horseshoe and rigidity theory for circle diffeomorphisms with breaks, Preprint IML-0910s-17 (2010).

[19] K. M. Khanin, E. B. Vul, Circle Homeomorphisms with Weak Discontinuities, Advances in Sov. Math. 3 (1991), 57-98.

[20] H. Koch and S. Kocić, Renormalization of vector fields and Diophantine invariant tori, Ergod. Theor. Dynam. Sys. 285 (2008), 1559-1585.

[21] H. Koch and S. Kocić, A renormalization group aproach to quasiperiodic motion with Brjuno frequencies, Ergod. Theor. Dynam. Sys. 30 (2010), 1131-1146.

[22] S. Kocić, Renormalization of Hamiltonians for Diophantine frequency vectors and KAM tori, Nonlinearity 18 (2005), 1-32.

[23] S. Kocić, Reducibility of skew-product systems with multidimensional Brjuno base flows, Discrete Contin. Dyn. Syst. 291 (2011), 261-283.

[24] M. Lyubich, Feigenbaum-Coullet-Tresser universality and Milnor's hairiness conjecture, Ann. Math. 1491 (1999), 319-420. 
[25] R. S. MacKay, J. D. Meiss and J. Stark, An approximate renormalization for the break-up of invariant tori with three frequencies, Physics Letters A 190 (1994), 417424 .

[26] S. Marmi, P. Moussa, J.-C. Yoccoz, Linearization of generalized interval exchange maps, Preprint arXiv:1003.1191 (2010).

[27] C. T. McMullen, Renormalization and 3-manifolds which fiber over the circle, Princeton University Press, Princeton, NJ, 1996.

[28] S. Ostlund, D. Rand, J. Sethna, E. Siggia, Universal properties of the transition from quasi-periodicity to chaos in dissipative systems, Physica 8D (1983), 303-342.

[29] Ya. G. Sinai, K. M. Khanin, Smoothness of conjugacies of diffeomorphisms of the circle with rotations, Uspekhi Mat. Nauk 441 (1989), 57-82.

[30] D. Sullivan, Bounds, quadratic diffrentials and renormalization conjectures, In American Mathematical Society centennial publications Vol. II (Providence, RI, 1988) 417-466; Amer. Math. Soc. (Providence, RI, 1992).

[31] M. Yampolsky, Hyperbolicity of renormalization of critical circle maps, Publ. Math. Inst. Hautes Etudes Sci. 96 (2002), 1-41.

[32] J.-C. Yoccoz, Conjugaison differentiable des difféomorphismes du cercle donc le nombre de rotation vérifie une condition Diophantienne, Ann. Sci. Ec. Norm. Sup. 17 (1984), 333-361. 